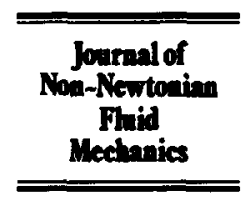

\title{
Dynamic simulation of sedimentation of solid particles in an Oldroyd-B fluid
}

\author{
J. Feng*, P.Y. Huang, D.D. Joseph \\ Department of Aerospace Engineering and Mechanics, University of Minnesota, Minneapolis, MN 55455, USA
}

Received 16 October 1995

\begin{abstract}
In this paper we present a two-dimensional numerical study of the viscoelastic effects on the sedimentation of particles in the presence of solid walls or another particle. The Navier-Stokes equations coupled with an Oldroyd-B model are solved using a finite-element method with the EVSS formalism, and the particles are moved according to their equations of motion. In a vertical channel filled with a viscoelastic fluid, a particle settling very close to one side wall experiences a repulsion from the wall; a particle farther away from the wall is attracted toward it. Thus a settling particle will approach an eccentric equilibrium position, which depends on the Reynolds and Deborah numbers. Two particles settling one on top of the other attract and form a doublet if their initial separation is not too large. Two particles settling side by side approach each other and the doublet also rotates till the line of centers is aligned with the direction of sedimentation. The particle-particle interactions are in qualitative agreement with experimental observations, while the wall repulsion has not been documented in experiments. The driving force for lateral migrations is shown to correlate with the pressure distribution on the particle's surface. As a rule, viscoelasticity affects the motion of particles by modifying the pressure distribution on their surface. The direct contribution of viscoelastic normal stresses to the force and torque is not important.
\end{abstract}

Keywords: Dynamic simulation; Oldroyd-B fluid; Sedimentation; Solid Particles

\section{Introduction}

When a flow phenomenon in a non-Newtonian fluid contrasts that which is expected in a Newtonian fluid, it is usually called an anomalous effect. As more and more such phenomena are discovered, people realize that "anomaly" is the rule. Such is the case with the motion of solid particles in viscoelastic liquids. In almost every case tested, the particle behaves qualita-

* Present address: Department of Chemical Engineering, University of California, Santa Barbara, CA 93106-5080, USA. 
tively differently in a non-Newtonian fluid. Earlier experimental findings have been summarized by Leal [1]. An elongated particle settling in an unbounded quiescent liquid rotates till its long axis aligns with the fall (Leal [2], Liu and Joseph [3]). A rod-like particle in a shear flow approaches a limiting orbit of rotation with its axis aligned with the vorticity vector (Karnis and Mason [4], Gauthier et al. [5,6]). In a Poiseuille flow, a particle migrates across streamlines to the center of the pipe $[4,6]$. Two spheres sedimenting one above another may attract or repel depending on their initial separations (Riddle et al. [7]).

Recently, experimental studies were carried out to investigate the effects of a nearby wall on a sedimenting sphere and the interaction between two spheres settling side by side (Joseph et al. [8]). In a Newtonian fluid, inertia is known to cause repulsion between the sphere and the wall and between the two spheres ( $\mathrm{Liu}$ et al. [9]). Not surprisingly, a complete reversal occurs in viscoelastic liquids; the sphere is pulled toward the wall and the two spheres attract if the initial separation is smaller than a critical value, beyond which no interaction is discernible. Tanner [10] noticed, in measuring the drag in a falling ball viscometer, that the ball tends to drift off center toward the wall under certain conditions. Jones et al. [11] recently reported similar observations in a rectangular column.

Highly successful perturbation theories were developed based on the second-order fluid model (Leal [1], Brunn [12]). Qualitatively correct predictions have been obtained for the preferred orientation of a settling long body [2], the lateral migration of a sphere in a non-homogeneous shear flow (Ho and Leal [13], Chan and Leal [14]) and the evolution of the Jeffery orbit [2]. The cause of various anomalous effects has been identified as normal stresses of the second-order fluid. As long as no wall-particle or particle-particle interactions are involved, the perturbation method yields satisfactory results. However, when Brunn [15] applied the same scheme to the interaction of two sedimenting spheres, results show that the two spheres always attract, in apparent disagreement with the observations of Riddle et al. [7]. Caswell [16] used a similar perturbation procedure to study the wall effects on the sedimentation of a sphere. His results show that the sphere would be repelled by the wall, again in contradiction to experimental observations. To summarize, perturbation theories apply well only to problems without wall or interaction effects. Its failure otherwise may be caused by the use of unrealistic constitutive laws.

Direct numerical simulation of the motion of solid objects in viscoelastic liquids has recently become possible. Most of the work in this area has been concerned with the ball-inside-cylinder geometry, inspired by the falling-ball viscometer. Effects of the Deborah number and wall blockage on the drag are of primary interest, and only steady flows are considered. A comprehensive review of these results can be found in Walters and Tanner [17]. Unsteady motion of solid particles causes two additional difficulties in computation: the transient effect in the fluid flow and the moving solid boundary. A number of authors have simulated the unsteady sedimentation of a ball along the center line of a vertical tube filled with a viscoelastic fluid (e.g., Bodart and Crochet [18], Becker et al. [19]). As far as we know, no other geometry or flow conditions have been studied for the transient motion of a particle.

In this paper, we present dynamic simulations of the sedimentation of particles in an Oldroyd-B fluid. The goal of this work is to study the fluid mechanics of wall-particle and particle-particle interactions. The algorithm is an extension of what we used in simulating moving boundary problems in Newtonian fluids [20,21]. At each time step, the equations of motion and the constitutive equation of the fluid are solved using a finite element solver 
POLYFLOW with an EVSS scheme (Debae et al. [22]). The force and torque on the solid particle then determine the position and velocity of the particle at the next time step. The new domain is re-meshed and the old velocity and stress fields are projected onto the new mesh. Then the velocity, pressure, stress and deformation gradient fields are computed for the new time. More details of the algorithm and the mesh are given by Huang and Feng [23].

As compared to its Newtonian counterpart, the viscoelastic code is more susceptible to numerical instabilities, and we frequently used refined mesh and reduced time increment to verify the convergence of the results. The highest Deborah number that can be handled by the code typically lies between 1 and 2 . The inability of lower-order EVSS methods to compute high Deborah number flows is well recognized (Khomami et al. [24]). This does not compromise our mission in this paper, however, because the leading-order effects of elasticity that we are interested in occur at low Deborah numbers already. We are currently limited to two-dimensional simulations. Nonetheless, this is the first attempt, to our knowledge, at dynamic simulation of particle motion in complex flows of viscoelastic fluids.

Two problems are studied in this paper: the sedimentation of a circular particle released in a vertical channel at various initial distances from the side wall and the sedimentation of a pair of particles released side by side or in tandem. Because the Newtonian counterparts of these problems have been studied [9,20], the effects of normal stresses can be highlighted. In Section 2 , we will first consider a few test problems and compare our numerical results with those of previous studies. This comparison serves as a validation of our program. Then numerical results for the two proposed problems will be discussed.

\section{Numerical results and discussions}

\subsection{Validation of the code}

We have found no data in the literature that affords a complete check of the validity of our code. Two previous studies seem to serve this purpose to some extent. Carew and Townsend [25] have computed the force and torque on a circular cylinder fixed at different eccentric positions in a creeping channel flow. A finite element method with a mixed Galerkin formulation is used. The fluid is represented by the Oldroyd-B model or the Phan-Thien-Tanner model. Their computation verified the lateral force that pulls the cyclinder toward the nearby wall, which was first measured by Dhahir and Walters [26] in experiments. This computation is in two dimensions like ours, and quantitative comparison is possible. Inertia of the fluid flow is, however, not included. Moreover, the flow is steady so the transient performance of our code cannot be tested. For this purpose, we use the axisymmetric computation of a sphere falling in a vertical tube $[18,19]$.

The geometry in Carew and Townsend [25] is shown in Fig. 1. The blockage ratio $2 a / L=$ $7 / 12$. The lateral position of the cylinder is indicated by an eccentricity factor

$$
e=1-d_{1} / d_{2} \text {. }
$$

Thus $e=0$ when the cylinder sits on the centerline of the channel; $e=1$ when the cylinder touches the wall. For an Oldroyd-B fluid 


$$
T+\lambda_{1} \stackrel{\nabla}{T}=2 \mu\left(D+\lambda_{2} \stackrel{\nabla}{D}\right)
$$

Carew and Townsend used $\lambda_{2} / \lambda_{1}=1 / 9$. A parabolic velocity profile is assigned to the inflow. Four positions of the cylinder have been computed: $e=0,0.33,0.57$ and 0.75 .

To compare with the results of Ref. [25], we set the inertia to zero in our program, and used exactly the same geometric and rheological parmeters. The length of the computational domain is $100 a$, about the same as in Ref. [25]. At the inlet of the domain, fully developed velocity and stress profiles are assumed. At the outlet, normal derivatives of the velocity are set to zero. We use an unstructured triangular mesh, which is much denser than the mesh in Ref. [25].

The drag, lift and torque on the cylinder are compared between the two computations in Fig. 2. The dimensionless force and torque coefficients are defined by

$$
\begin{aligned}
& C_{\mathrm{d}}=\frac{\text { Drag per unit length }\left(F_{x}\right)}{\mu U_{0}}, \\
& C_{\mathrm{l}}=\frac{\text { Lift per unit length }\left(F_{y}\right)}{\mu U_{0}}, \\
& C_{\mathrm{t}}=\frac{\text { Torque per unit length }\left(T_{z}\right)}{\mu U_{0} a} .
\end{aligned}
$$

Counter-clockwise torque is defined as positive. We define a Deborah number

$$
D e=U_{0} \lambda_{1} / a,
$$

which differs from that of Carew and Townsend [25] by a factor of $\left(1-\lambda_{2} / \lambda_{1}\right)$. Their data have been converted to our $D e$ in Fig. 2.

Both studies concur that there is a drag reduction with increasing De (Fig. 2(a)). The Newtonian drag agrees fairly well, but Carew and Townsend [25] obtained a much smaller drag for viscoelastic fluids. For eccentric positions, both computations predict a wall attraction force (Fig. 2(b)). This force increases with the Deborah number; for a fixed $D e$, it has a maximum at some intermediate position between $e=0.33$ and 0.75 . The magnitude of this attraction force is about $50 \%$ larger in our calculation. Both computations yield a positive torque on the cylinder (Fig. 2(c)), which increases monotonically in our calculation but has a minimum at $D e \approx 0.12$ in Ref. [25].

In summary, our EVSS formulation gives qualitatively the same results as Carew and Townsend [25], but considerable numerical discrepancies exist. This is perhaps owing to the coarse mesh used in Ref. [25]. Their finest mesh has only 112 elements. This was shown to be sufficient for Newtonian flows; no convergence test was given for viscoelastic flows. Our

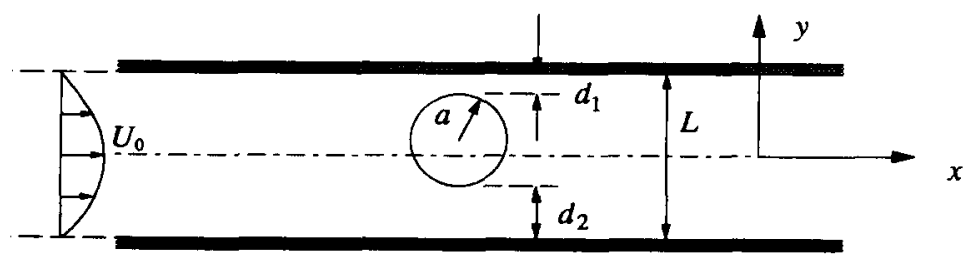

Fig. 1. Geometry of the channel flow around a fixed cylinder computed by Carew and Townsend [25]. 
numerical experiments indicate that viscoelastic flows generally require much more refined mesh. Our typical mesh has 500 elements and 1100 grid points.

The settling of a sphere or cylinder in a tube or plane channel filled with Oldroyd-B fluid (Eq. (2)) is determined by the following dimensionless groups:
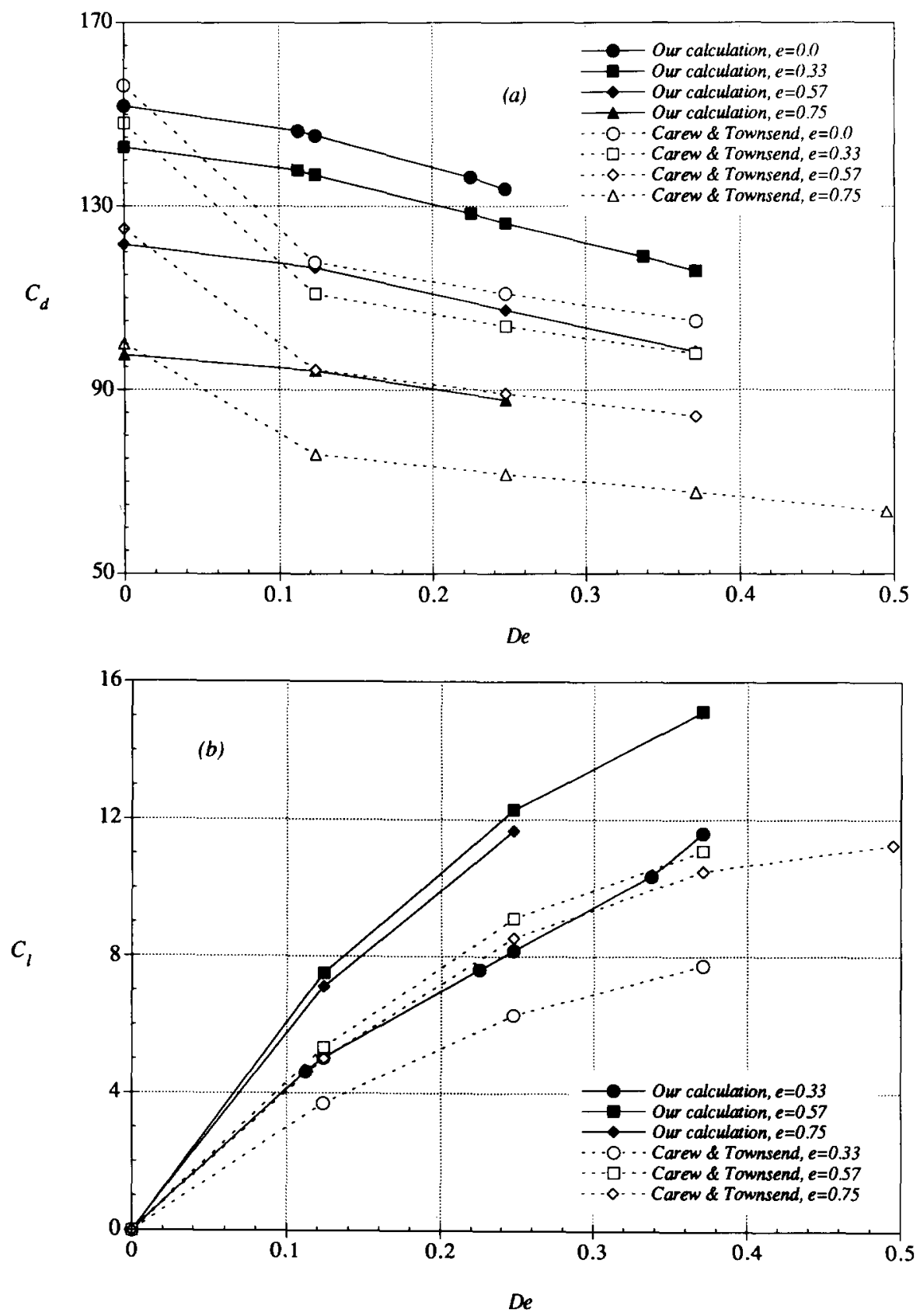

Fig. 2. (a), (b). 


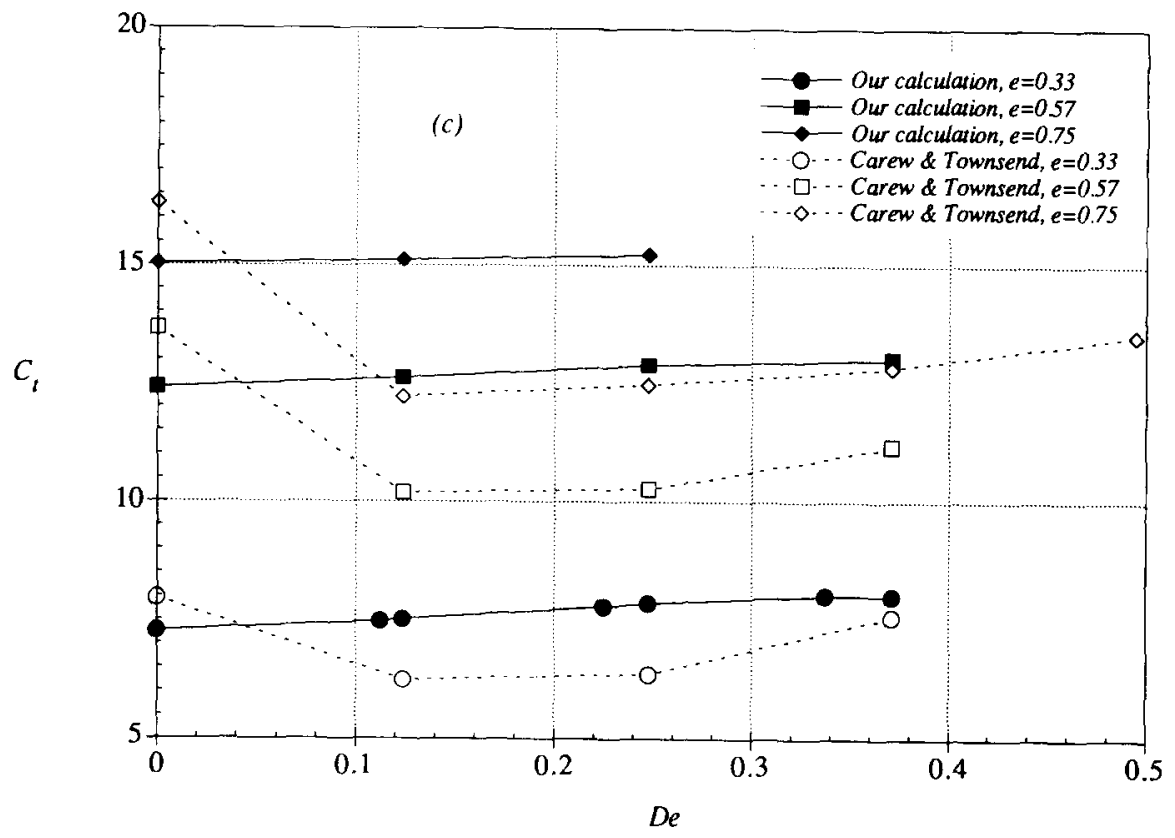

Fig. 2. Comparison between our numerical results and those of Carew and Townsend [25]: (a) drag, (b) lift, (c) torque.

$$
R e=\frac{\rho_{\mathrm{f}} U_{\mathrm{t}} a}{\mu}, \quad D e=\frac{U_{\mathrm{t}} \lambda_{1}}{a}, \quad F r=\frac{g a}{U_{\mathrm{t}}^{2}}, \quad \frac{a}{R}, \quad \frac{\rho_{\mathrm{s}}}{\rho_{\mathrm{f}}}, \quad \frac{\lambda_{2}}{\lambda_{1}},
$$

Where $U_{\mathrm{t}}$ is the terminal velocity of the particle and $R$ is the radius of the tube or the semiwidth of the channel. In comparing our planar case with axisymmetric cases, it is obviously impossible to match all six parameters. Thus, a meaningful quantitative comparison cannot be made. Fig. 3 compares the variation of the settling velocity obtained in our calculation with two axisymetric calculations for spheres. Bodart and Crochet [18] used a mixed finite-element method with a Galerkin formulation. The mesh is attached to the sphere and translates with it. Becker et al. [19] used a Lagrangian finite element method with a single integral Oldroyd-B model. A large overshoot in the falling speed is obtained in all three computations, consistent with the experimental observations of Walters and Tanner [17] and Becker et al. [19]. The terminal velocity is approached through a damped oscillation.

The above comparisons indicate that our code gives qualitatively reasonable results for the two test problems. Its accuracy cannot be assessed at present owing to lack of comparable data.

\subsection{Sedimentation of a single particle in a channel}

The geometry of the problem is shown in Fig. 4. A cylinder of radius $a$ and density $\rho_{\mathrm{s}}$ is released at an eccentric position in a channel filled with an Oldroyd-B fluid. The cylinder is heavier than the fluid and starts to settle under gravity. We wish to study the lateral motion of the particle as a result of the walls.

Before doing dynamic simulations of the sedimentation, we first carried out static calculations. The particle is fixed in space; a uniform velocity $U$ is applied at the inlet of the domain and on 


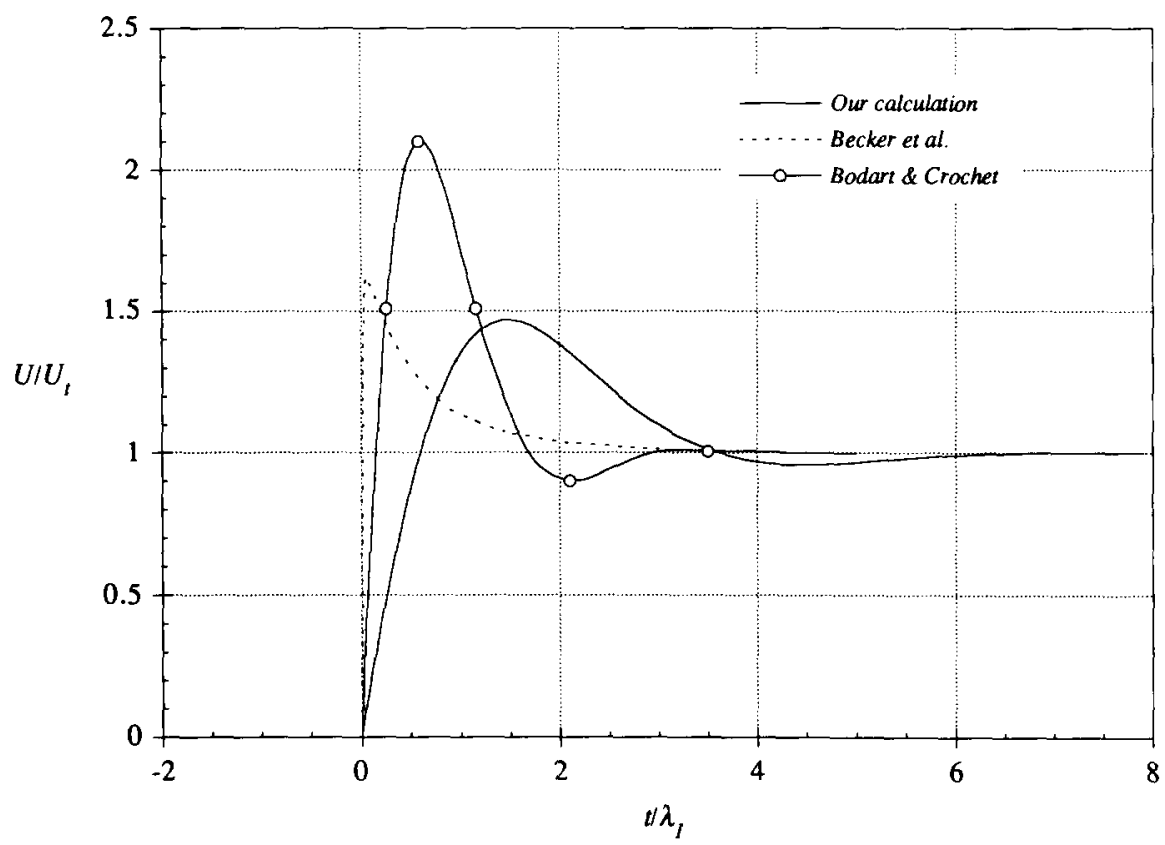

Fig. 3. Comparison of the settling velocity in three studies. Our calculations: $R e=0.02255, D e=0.2255, F r=10.36$, $a / R=0.5, \rho_{\mathrm{s}} / \rho_{\mathrm{f}}=26.74, \lambda_{2} / \lambda_{1}=1 / 9$. Bodart and Crochet [18]: $R e=0.6468, D e=1.986, F r=4.976, a / R=0.5$, $\rho_{\mathrm{s}} / \rho_{\mathrm{f}}=7.162, \lambda_{2} / \lambda_{1}=1 / 9$. Becker et al. [19] $R e=5.27 \times 10^{-3}, D e=0.402, a / R=0.243$. Other parameters are not given.

both side walls. This is a Galilean transformation of a steady sedimentation without lateral migration and rotation. The lateral force on the particle then suggests the direction of lateral motion if the constraints are removed. The static calculations are important for two reasons. Firstly, by placing the cylinder at different lateral positions, we learn where interesting behaviors are expected. Then we can use these as initial positions in dynamic simulations. Secondly, effects of various parameters such as the Reynolds number and the Deborah number can be explored using static calculations. Both considerations are based on the fact that dynamic runs usually

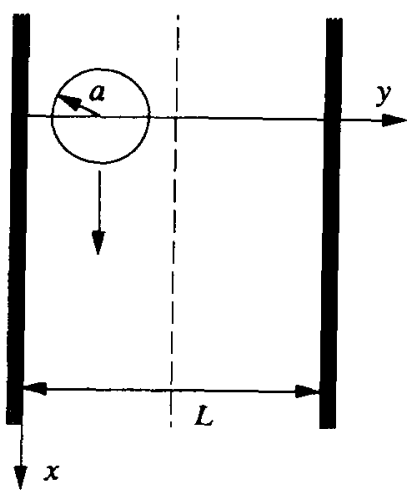

Fig. 4. Sedimentation of a single cylinder in a channel filled with an Oldroyd-B fluid. 


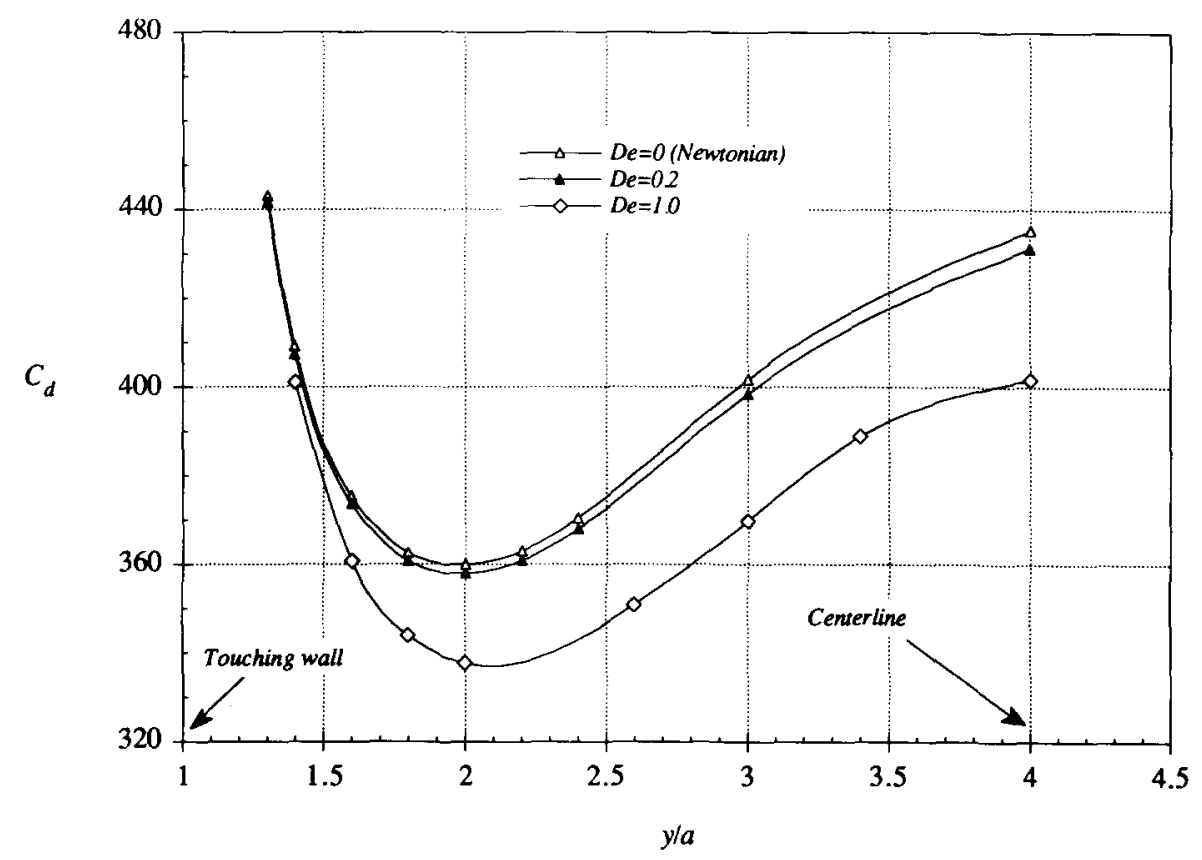

Fig. 5. The drag on a cylinder at different positions. $R e=0.05, L / a=8$.

have long transients and require tremendous amount of computation. With the help of static calculations, we may gain a complete picture of the sedimentation by doing just a few dynamic simulations. Next, we will present the results of static calculations first. Then several dynamic simulations are discussed. Finally, we explore the origin of the cylinder's behavior by analyzing the pressure distribution around its surface.

\subsubsection{Static calculations}

We use an Oldroyd-B model with $\lambda_{2} / \lambda_{1}=1 / 8$. The control parameters are $R e=\rho_{\mathrm{f}} U a / \mu$, $D e=U \lambda_{1} / a$ and the blockage ratio $L / a$. We define a drag coefficient $\left(C_{\mathrm{d}}\right)$ and a lift coefficient $\left(C_{l}\right)$ by dividing the forces by $\left(\rho_{f} U^{2} a\right)$, and a torque coefficient $\left(C_{t}\right)$ by dividing the torque by $\left(2 \rho_{\mathrm{f}} U^{2} a^{2}\right)$.

Results show that at relatively low Reynolds numbers, the drag has a minimum when the cylinder is somewhere between the centerline and the wall (Fig. 5). For $L / a=8$, this minimum occurs at $y / a \approx 2$. The minimum drag is consistent with the creeping flow results of Dvinsky and Popel [27]. As the Deborah number is increased, the drag is reduced. The torque on the cylinder is positive for $y<L / 2$ (Fig. 6), meaning that the cylinder would rotate as if rolling up the nearby wall. Increasing $D e$ tends to reduce the magnitude of this torque.

The variation of the lateral force is much more intriguing. Typically, there is a region near the wall in which strong wall repulsion prevails. Between this wall region and the centerline of the channel is a core region in which the cylinder experiences attraction toward the nearby wall. The effect of $D e$ is to promote wall attraction (Fig. 7). At $D e=0$, a wall repulsion prevails throughout the entire region, a well-known fact from previous study of Newtonian flows [20]. As 
$D e$ increases from 0.2 to 1 , the core region becomes wider and the magnitude of the attraction force is greatly increased. The wall region is narrowed, though the repulsion force sees an increase. The effect of $R e$, on the other hand, is to suppress the wall attraction (Fig. 8). As compared with the creeping flow $(R e=0, D e=0.2)$, the inertial flow at $R e=0.05$ and $D e=0.2$ has a smaller core region with a weaker attraction force and a stronger repulsion in the wall region. It is interesting to see how the lift force and the two regions change when $R e$ and $D$ e are varied simultaneously while the ratio $D e / R e$ is kept constant. This corresponds to a series of experiments using particles of the same diameter but different densities in the same fluid. For $D e / R e=4$, the core region dwindles only slightly as $D e$ and $R e$ are increased (Fig. 9). Inside the core region, the wall attraction becomes weaker, suggesting that the inertia is winning over the normal stress. In the wall region, a large increase in the repulsion force is observed since both effects promote this repulsion.

The geometric parameter $L / a$ has an unexpectedly strong effect on the lateral force. Narrower channels have a relatively wider core region with a larger attraction force (Fig. 10). To compare the lift curves in different channels, we use the eccentricity factor $e$ (eq. (1)). For $L / a=3.43$ (the aspect ratio used in Ref. [25]), the core region extends to $e=0.92$. On the other hand, no core region is observed for a wide channel of $L / a=20$; wall repulsion prevails throughout.

The above results imply that the wall attraction is not a result of a single wall, but rather is caused by two walls. This is different from the concept of wall attraction derived from experiments $[8,11]$. There are two possible explanations for this apparent discrepancy: (i) our numerical results are valid in two dimensions only, and the wall effects are markedly different in two and three dimensions; or (ii) the present results are qualitatively valid in three

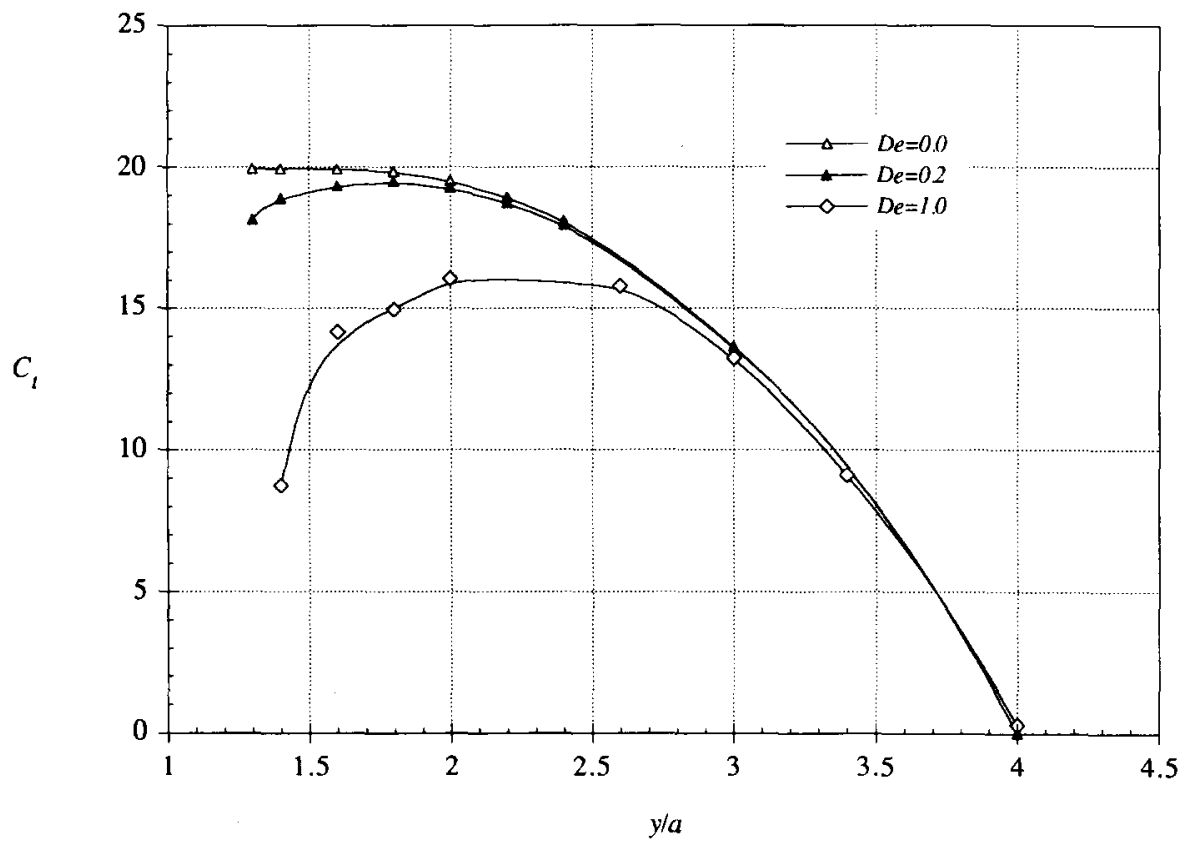

Fig. 6. The torque on a cylinder at different positions. $R e=0.05, L / a=8$. 


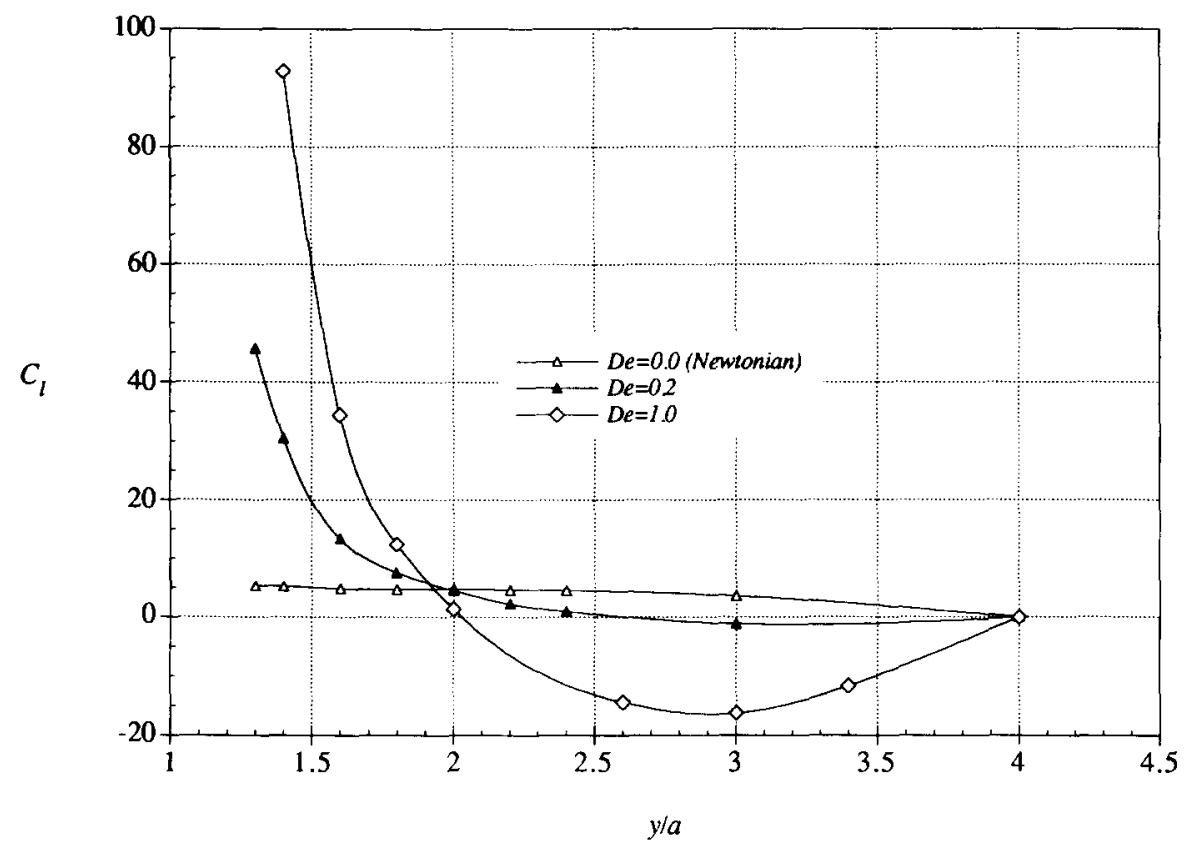

Fig. 7. The lift on a cylinder at various lateral positions. $R e=0.05, L / a=8$. The Deborah number tends to promote the attraction force.

dimensions, and the previous notion of attraction toward a single wall is misguided. Wang, Feng and Joseph [28] carried out a three-dimensional calculation is an enclosed domain. A perturbation scheme is used to extract the leading-order viscoelastic effects on the lateral force between a sphere and a nearby wall. A repulsion force is obtained in a region next to the wall and an attraction force exists further out. This result appears to be evidence, for (ii). But it remains to be seen whether the core region will dwindle or even disappear when the other walls are moved away (see Fig. 10). One may also argue that no experiment can realise the flow conditions in a truly semi-infinite domain; other side walls are always present to enclose the body of fluid. If the Deborah number is large and the Reynolds number is small, the wall attraction will dominate the entire region except for a thin wall layer that is perhaps difficult to detect experimentally. Indeed, Joseph et al. [8] noticed that under certain conditions, a sphere does not come into contact with the wall but rather keeps a small stand-off distance. In addition, the numerical results in Figs. 7 and 10 seem to enjoy support from the falling-ball experiment of Tanner [10]. A critical ball-cylinder radius ratio can be defined. Smaller balls fall along the center of the cylinder. Once the radius ratio exceeds the critical value, about 0.21 for the viscoelastic solution used, the ball drifts off the center and assumes an eccentric position, rotating meanwhile in the sense opposite to that of a sphere rolling down the nearby wall. This observation is consistent with the effects of $L / a$ obtained in our computation. To summarize, the apparent discrepancy between numerical and experimental results cannot be resolved unambiguously based on our current knowledge. More rigorous comparison between two- and three-dimensional data is needed. 
Some liquids used in the experiments are shear-thinning. To test this effect, we used the Carreau-Bird viscosity function in our calculations (see Ref. [23]). The power index $n=1$ corresponds to no shear-thinning, and smaller $n$ indicates more shear-thinning. The curve with $n=0.2$ in Fig. 8 shows that shear-thinning causes a small decrease in the magnitude of the lateral force in the wall and core regions alike. This does not seem to account for the discrepancy between numerics and experiments discussed above.

\subsubsection{Dynamic simulations}

The transient sedimentation of particles depends on the Froude number $F r=g a / U_{\mathrm{t}}^{2}$ and the density ratio $\rho_{\mathrm{s}} / \rho_{\mathrm{f}}$ besides $R e$, De and $L / a$. The dynamic behavior of a settling particle is manifest of the wall-particle interaction that we have learned from static calculations. Fig. 11 shows the sedimentation of a particle released from different initial positions. An equilibrium position at $y / a=2.76$ is reached by all trajectories. The effect of the Deborah number is depicted in Fig. 12. The curves were obtained by varying the relaxation time of the fluid $\lambda_{1}$ while keeping other parameters fixed. Because the drag on the particle depends on De [23], the terminal velocity $U_{\mathrm{t}}$ and thus $R e$ and $F r$ also change. It is clear that larger $D e$ results in an equilibrium position closer to the channel wall, indicating a wider core region in which wall attraction prevails (see Fig. 7). In narrower channels, a particle stabilizes closer to the wall, consistent with Fig. 10. For $L / a=3.43$, a particle released near the center migrates all the way to the wall; the equilibrium position is such that the particle almost touches the wall (Fig. 13).

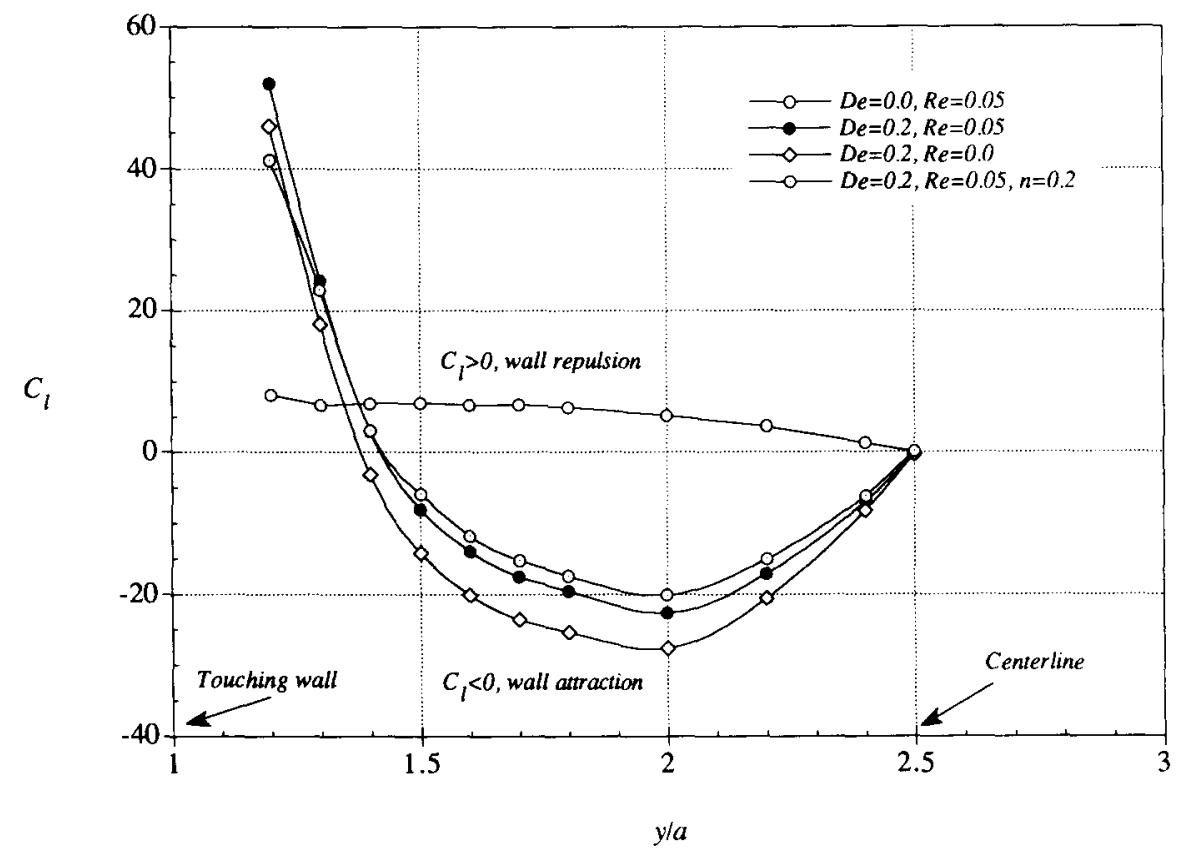

Fig. 8. The lift on a cylinder at various lateral positions. $L / a=5$. The Reynolds number tends to suppress the attraction force. A curve for Newtonian flow is also shown for comparison. The curve with $n=0.2$ indicates the effects of shear-thinning on the lateral force (discussion at the end of Section 2.2.1.). 


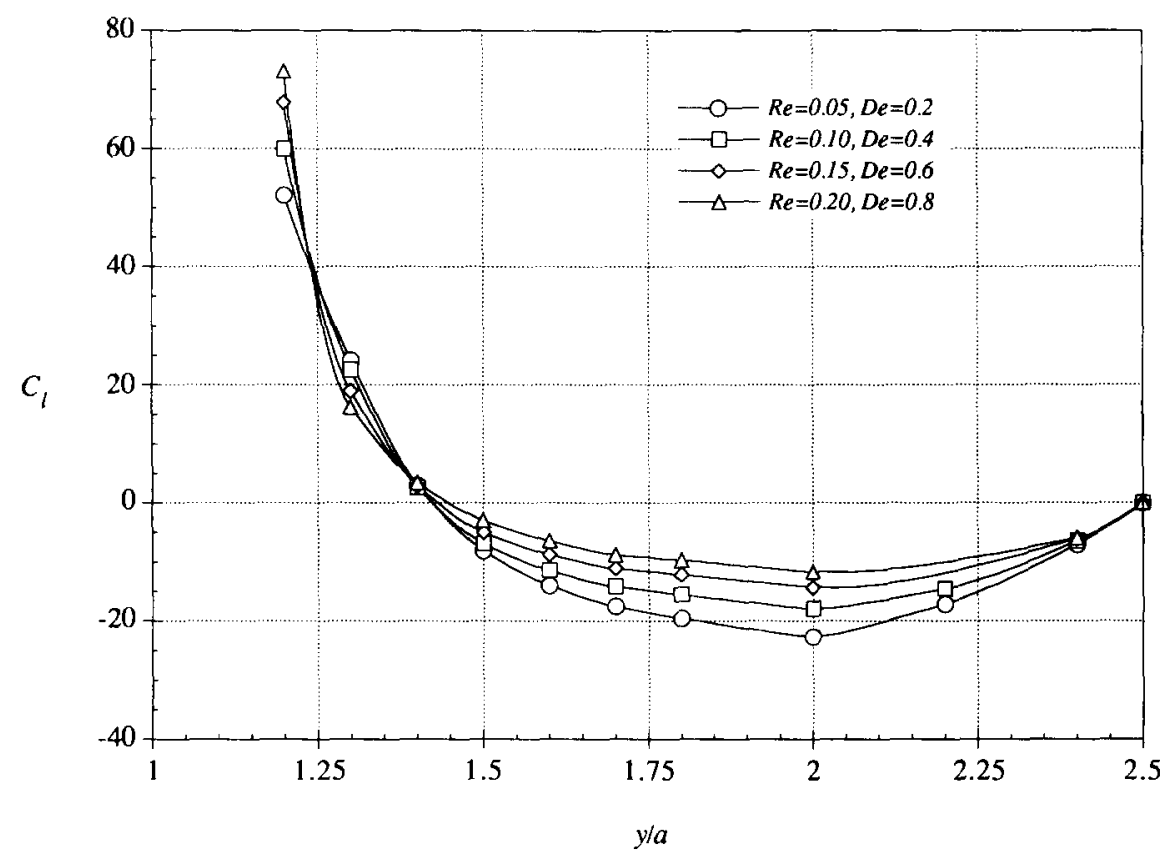

Fig. 9. Variation of the lift curve when $R e$ and $D e$ are varied while $D e / R e$ is kept constant. $L / a=5$.

\subsubsection{Stress analysis}

In the above discussions, viscoelasticity is shown to generate a wall repulsion on the particle in the wall region and an attraction in the core region. In previous works on the motion of solid particles in Newtonian and non-Newtonian fluids [21,29], we analyzed the forces and torque related to inertia or non-Newtonian rheology by studying the stress distribution on the surface of the particle. Here the same scheme will be followed. Static results will be used because the lateral motion in dynamic runs is accompanied by a viscous drag that in part obscures the driving force due to viscoelasticity.

In the EVSS formulation used here, the total stress tensor may be written as

$$
T=-p I+2 \mu D+S,
$$

Where $p$ is pressure, $\boldsymbol{D}$ is the rate-of-deformation tensor and $\boldsymbol{S}$ is an extra-stress tensor. It is easy to show that the flow next to the surface of the particle is a simple shear flow except for two stagnation points. Thus, for an Oldroyd-B fluid, the shear stress on the surface comes entirely from the $2 \mu \boldsymbol{D}$ term, and $S$ only contributes to the normal stress. Therefore, the force on the particle is a summation of three integrals: an integral of the pressure, that of the shear stress (from the viscous term) and that of the elastic normal stress. The projections of these integrals in the lateral direction are denoted by $P, V$ and $E$. Numerical results show that in all cases computed, pressure makes by far the largest contribution to the lateral force. For example, $P: E: V \approx 5: 1: 0.28$ at $R e=0.05, D e=0.2, L / a=5$ and $y / a=2$ (see Fig. 8). Closer to the wall at $y / a=1.2$, we have $P: E: V \approx 18: 1: 1.6 \times 10^{-2}$, with still stronger pressure domination. This means that viscoelasticity generates the lateral force by modifying the pressure distribution; the direct contribution of the normal stresses is rather small. The same behavior has been observed in a 
three-dimensional perturbation study of the slow sedimentation of an ellipsoid in a second-order fluid [29]. Hence, the effects of viscoelasticity can be examined by comparing the pressure distributions for Newtonian and viscoelastic fluids.

Fig. 14 shows pressure distributions on a circular particle located at $y / a=2$ in a channel of width $L=5 a$. The pressure coefficient $C_{\mathrm{p}}$ is defined by

$$
C_{\mathrm{p}}=\frac{p}{\frac{1}{2} \rho_{\mathrm{f}} U^{2}} .
$$

For the Newtonian case $(R e=0.05, D e=0)$, the high pressure at the front stagnation point $\left(\theta \approx 200^{\circ}\right)$ gives rise to a weak repulsion force that pushes the particle away from the wall. (A more detailed analysis of the repulsion due to inertia can be found in Ref. [9]). For the viscoelastic case $(D e=0.2)$, the pressure is elevated on the two sides of the cylinder $\left(\theta=90^{\circ}\right.$ and $\left.\theta=270^{\circ}\right)$ and decreased on the top and the bottom $\left(\theta=0^{\circ}\right.$ and $\left.\theta=180^{\circ}\right)$. This trend is more obvious at higher Deborah numbers. The pressure increase on the right side of the cylinder (near $\theta=90^{\circ}$ ) is larger than that on the left side (near $\theta=270^{\circ}$ ), which is closer to the wall. This imbalance gives rise to the wall attraction. Fig. 14 represents yet another example of the antagonism between inertia and non-Newtonian normal stress. We also note that the modification of pressure by viscoelasticity agrees with our three-dimensional perturbation study [29]. In that calculation, viscoelastic effects are shown to create high pressures on the left and right of a settling ellipsoid and low pressures on the top and bottom. This is just opposite to the inertial effects; this pressure distribution generates a torque that turns the ellipsoid to a long-axis vertical posture.

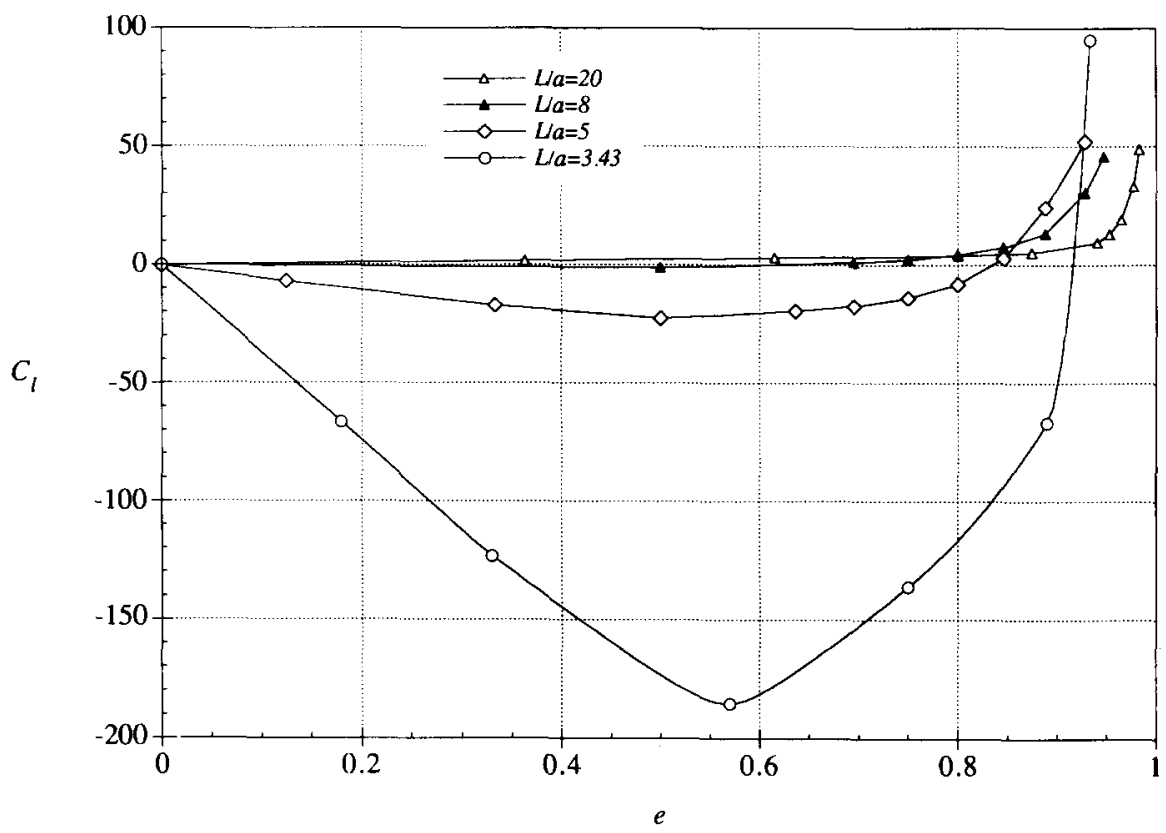

Fig. 10. Effects of the blockage ratio $L / a$ on the lift force. $R e=0.05, D e=0.2$. The eccentricity factor $e=0$ on the center line; $e=1$ when the cylinder touches one side-wall. 


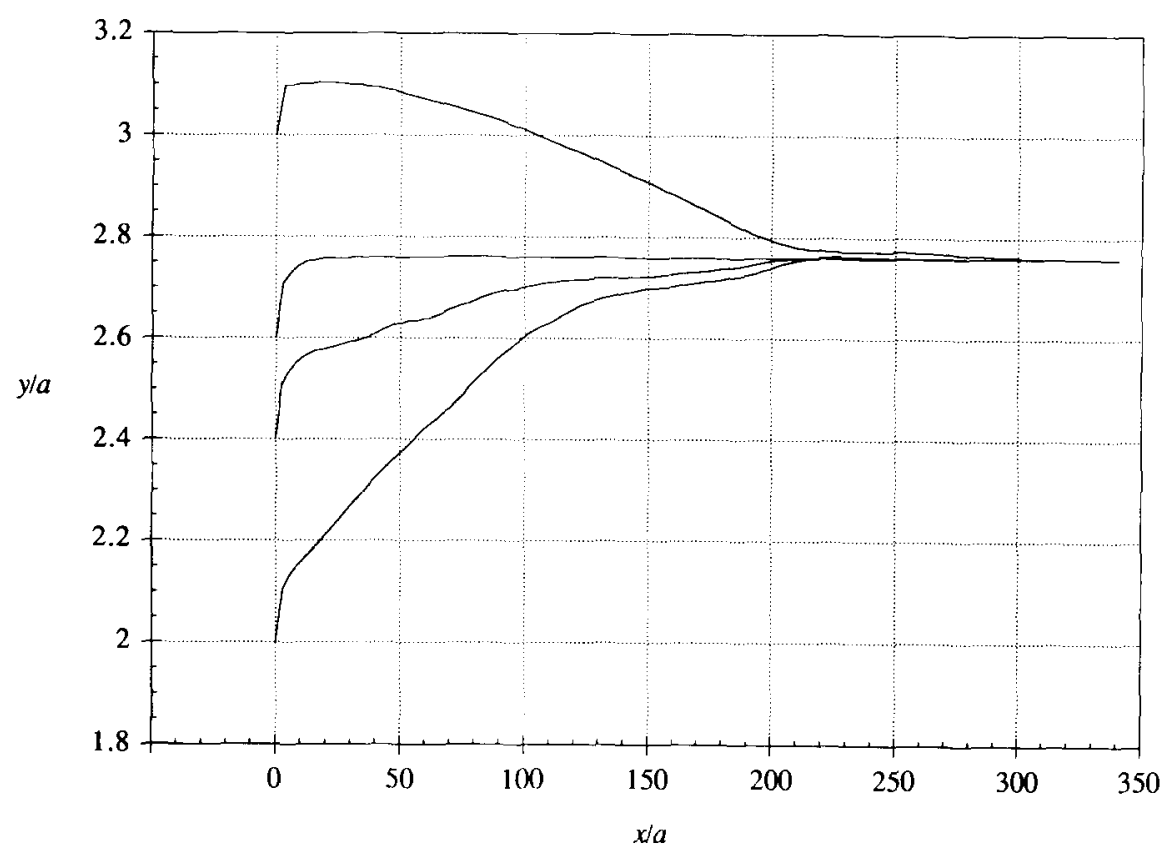

Fig. 11. Sedimentation of a particle released from different initial positions. $L / a=8, \rho_{\mathrm{s}} / \rho_{\mathrm{f}}=1.0007, R e=0.22$, $D e=0.97, F r=2.53 \times 10^{4}$.

For a particle placed very close to the wall, a different picture is obtained (Fig. 15). The high shear-rate in the narrow passage near $\theta=270^{\circ}$ leads to a very large pressure gradient. The Newtonian pressure distribution again features a large stagnation pressure between $\theta=180^{\circ}$ and $\theta=270^{\circ}$, which gives rise to an inertial repulsion. The non-Newtonian effects again cause an increase of pressure on the two sides of the circle. This time, however, the pressure increase is stronger on the near-wall side (near $\theta=270^{\circ}$ ), and this gives rise to a strong wall repulsion. Based on the current information, we are unable to explain how the wall proximity in Fig. 15 reverses the imbalance of pressure between the left and right sides of the particle.

\subsection{Interaction of two particles settling in a channel}

Brunn [15] used a perturbation method to study the interaction between two spheres settling far from each other through a second-order fluid. Results show that regardless of their relative positions, the spheres attract and approach each other while the line of centers turns to the vertical direction. This prediction is qualitatively correct for two spheres falling side by side [8]. Two spheres falling tandem, however, repel each other when they are far apart [7]. By using numerical simulations, we are rid of the restrictions on slow flow and large separations between particles.

\subsubsection{The vertical configuration}

Riddle et al. [7] observed in experiments that two spheres released tandem in a viscoelastic liquid will attract and come into touch if their initial separation is below a critical value that 
depends on the rheological properties of the fluid. If the initial separation exceeds this critical value, the two spheres will become farther apart during the settling.

We study the particle-particle interactions by static and dynamic simulations. In the static calculations, two circular particles of radius $a$ are fixed on the centerline of a plane channel of width $L=8 a$; the channel is filled with an Oldroyd-B fluid with $\lambda_{1} / \lambda_{2}=1 / 8$. The center-to-center distance between the particles is $s$. In this computation, the crucial factor is the drag forces on both particles. If the drag on the particle on top $\left(D_{1}\right)$ is larger than that on the particle at the bottom $\left(D_{2}\right)$, the two will seperate during sedimentation. Contrarily, the particle on top will catch up with the bottom one if $D_{2}>D_{1}$. Fig. 16 shows the variation of the drag coefficient at different separations. $C_{\mathrm{d}}, R e$ and $D e$ are defined as in Fig. 5. In the Newtonian case, the drag is essentially the same on the two particles. This is because the inertia is small at $R e=5 \times 10^{-3}$. At Deborah number $D e=1$, particle 1 (on top) experiences a smaller drag than particle 2 if the particles are fairly close $(s / a<7)$. In the range $7<s / a<9$, there is a reversal in the relative magnitude of the drag; the bottom particle has a slightly smaller drag. At $s / a=10$, the two drag forces are almost identical, indicating negligible interactions between particles at this separation or farther apart. Apparently, the static results are consistent with the experimental observations of Riddle et al. [7]. One may also note in Fig. 16 that the introduction of viscoelasticity tends to reduce the drag on both particles; this is true except when the two are very close, in which case the particle at the bottom acquires a larger drag than its counterpart in a Newtonian fluid.

Dynamic simulations are done for inertialess flows $(R e=0)$. This is in part because the behaviour to be simulated has nothing to do with inertia, as is obvious from Fig. 16. This is also the case in the experiment of Riddle et al. [7]. Since $R e$ is not directly controlable in the computation, it is troublesome to ensure that the fluid inertia, if retained, is not obscuring the desired effect. An

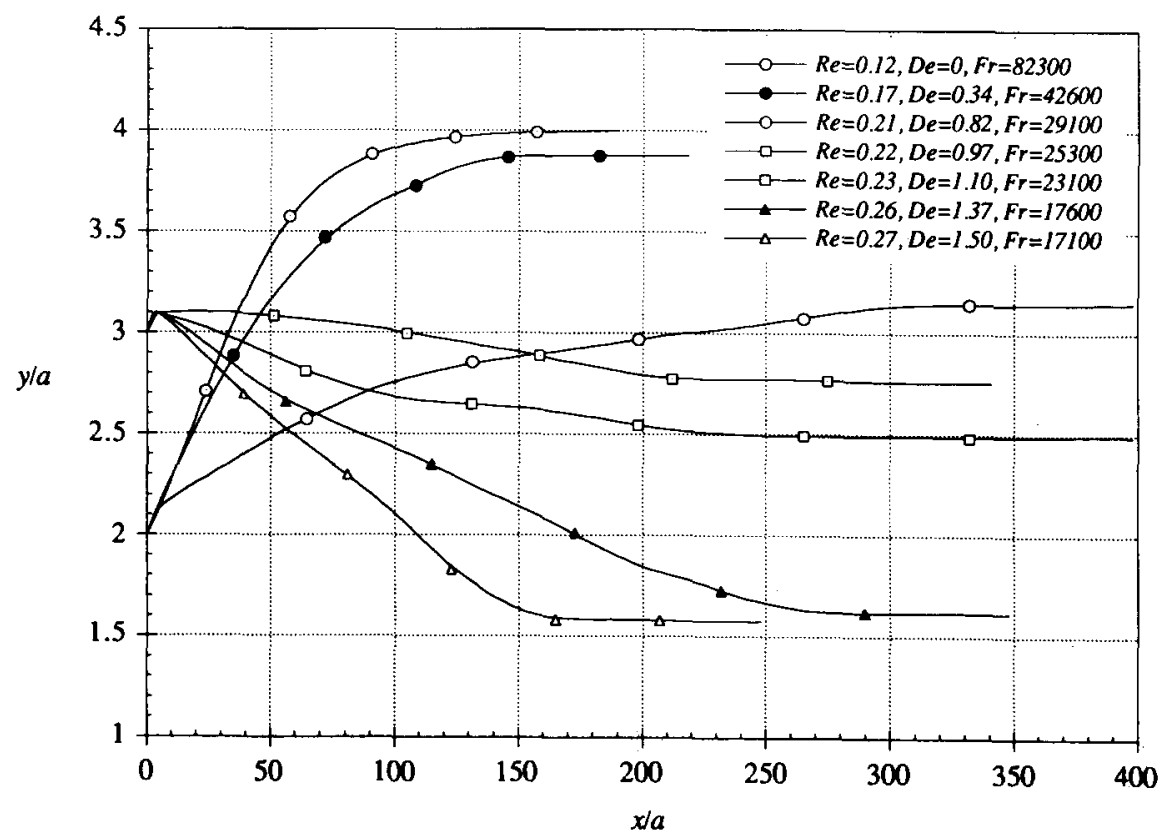

Fig. 12. The effect of viscoelasticity on the trajectory of a particle settling in a channel. $L / a=8, \rho_{\mathrm{s}} / \rho_{\mathrm{f}}=1.0007$. $R e$, $D e$ and $F r$ all change as the relaxation time of the fluid $\lambda_{1}$ is changed. 


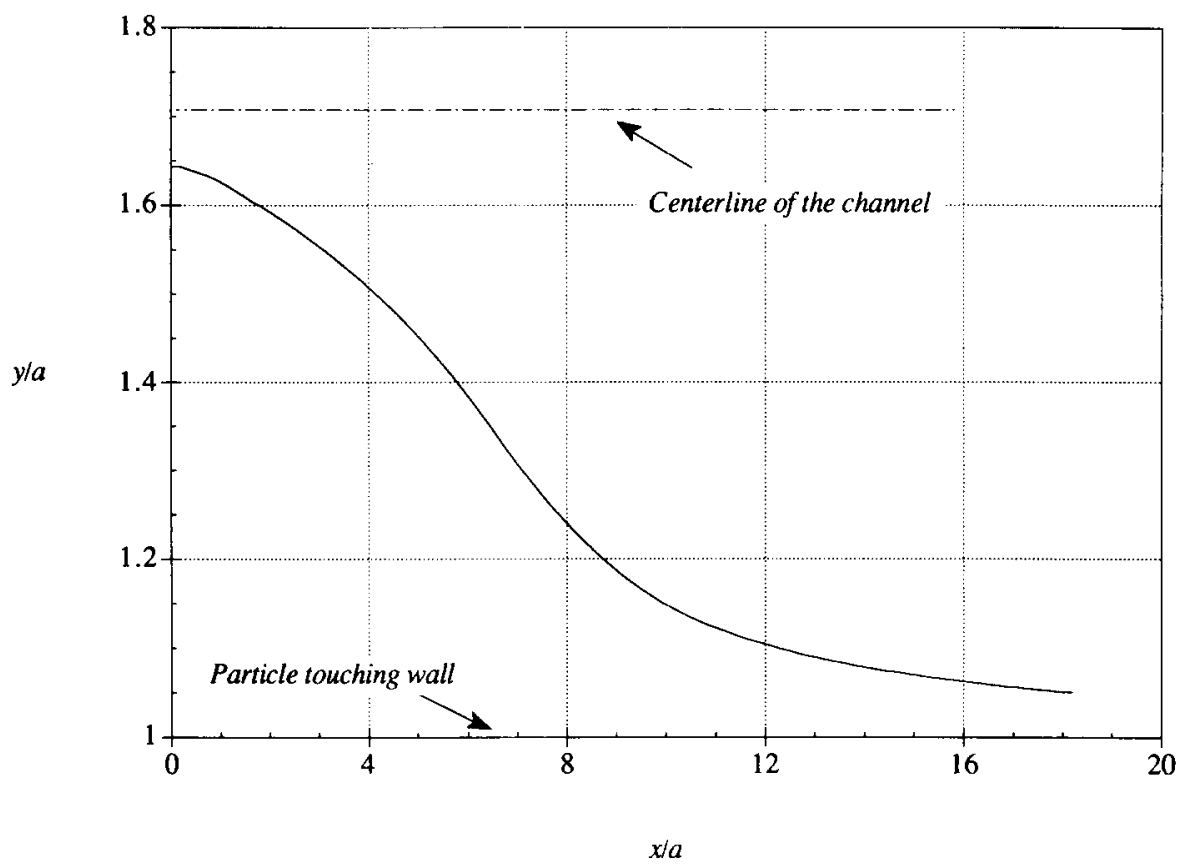

Fig. 13. A particle is attracted toward the wall in a narrow channel. $L / a=3.43, \rho_{\mathrm{s}} / \rho_{\mathrm{f}}=1.00075, R e=0.092$, $D e=0.37, \mathrm{Fr}=1.44 \times 10^{5}$.

additional benefit of neglecting fluid inertia is reduced computational cost; the non-linear inertia requires a Newton iteration. The inertia of solid particles is retained.

Three dynamic runs are shown in Fig. 17. The particles are released at different initial separations: $s_{0}=4 a, 8 a$ and $10 a$. In the first case, the particle on top rapidly catches up with the other. When they become too close to each other, the simulation breaks down. In reality the particles will come into contact and fall as a doublet [7]. The other two runs are intended to demonstrate the weak repulsion force found in static calculations. This scenario turns out to be difficult to realize dynamically. For $s_{0}=8 a$, the center-to-center distance $s$ does tend to grow at the beginning. But this trend is quickly reversed, and the particles start to get closer slowly. For $s_{0}=10 a, s$ never increases; it maintains its initial value for a short time and then starts to decrease. Finally $s$ seems to attain an equilibrium value at $9.85 a$. The "terminal" velocity is virtually the same in the last two cases; the doublet formed in the first case $\left(s_{0}=4 a\right)$ falls much faster.

The failure to simulate the separation of particles is probably a result of the transient nature of the motion. During sedimentation, the velocities of the particles change constantly. This has two effects on the relative magnitude of the drag. Firstly, because of the unsteadiness, the drag forces will be different from those in a steady flow at the same velocity. Secondly, the Deborah number and Reynolds number based on the instantaneous velocity are different from the projected terminal values. Then the ranges of $s / a$ corresponding to attraction and repulsion (see Fig. 16) may also be different. Therefore, the dynamic behavior of a pair of particles in sedimentation cannot be safely predicted from static forces based on their initial separation and 
the projected terminal velocity. Besides, the repulsion is very weak in our two-dimensional calculation and thus easily drowned out in dynamic simulation. The situation seems to be different in three dimensions: the separation of spheres is a fairly robust feature in Ref. [7].

The particle-particle attraction can be traced to special pressure distributions on the particles produced by viscoelasticity. We pick the configuration with $s=3 a$ in Fig. 16 for analysis. In both Newtonian and viscoelastic calculations, pressure and viscous shear stress make comparable contributions to the drag on each particle. The contribution of elastic normal stresses is at least one order of magnitude smaller. More interestingly, the viscous part of the drag differs by $3 \%$ between the two particles at $D e=1$, whereas the pressure part differs by $30 \%$. Hence, the following rule is again observed: viscoelastic effects are realized through a modified pressure field, and the direct contribution of normal stresses is unimportant.

Fig. 18(a) shows the pressure distributions on the particles in a Newtonian fluid. The pressure at the midpoint between the particles is set to zero. $C_{\mathrm{p} 1}$ and $C_{\mathrm{p} 2}$ are essentially mirror images of each other, indicating negligible inertia. Viscoelasticity breaks this symmetry (Fig. 18(b)). The pressure on the two sides of the particles (near $\theta=90^{\circ}$ and $270^{\circ}$ ) is elevated, consistent with previous results of perturbation [29]. The dashed line in the plot is a mirror image of $C_{\mathrm{p} 2}$, which is what $C_{\mathrm{p} 1}$ would be to maintain the symmetry. The difference between $C_{\mathrm{p} 1}$ and $-C_{\mathrm{p} 2}$ is larger in the first and fourth quadrants $\left(0^{\circ}<\theta<90^{\circ}\right.$ and $\left.270^{\circ}<\theta<360^{\circ}\right)$ than in the second and third $\left(90^{\circ}<\theta<270^{\circ}\right)$. The consequence is a downward net force on particle 1 , whose magnitude equals the difference in drag between the particles. Alternatively, one may compare $C_{\mathrm{p} 2}$ and $-C_{\mathrm{pl}}$ and argue that particle 2 experiences an upward force. The weak repulsion extant for larger $s$ is not easily borne out by pressure distributions, and stress analysis will not be done for the repulsion.

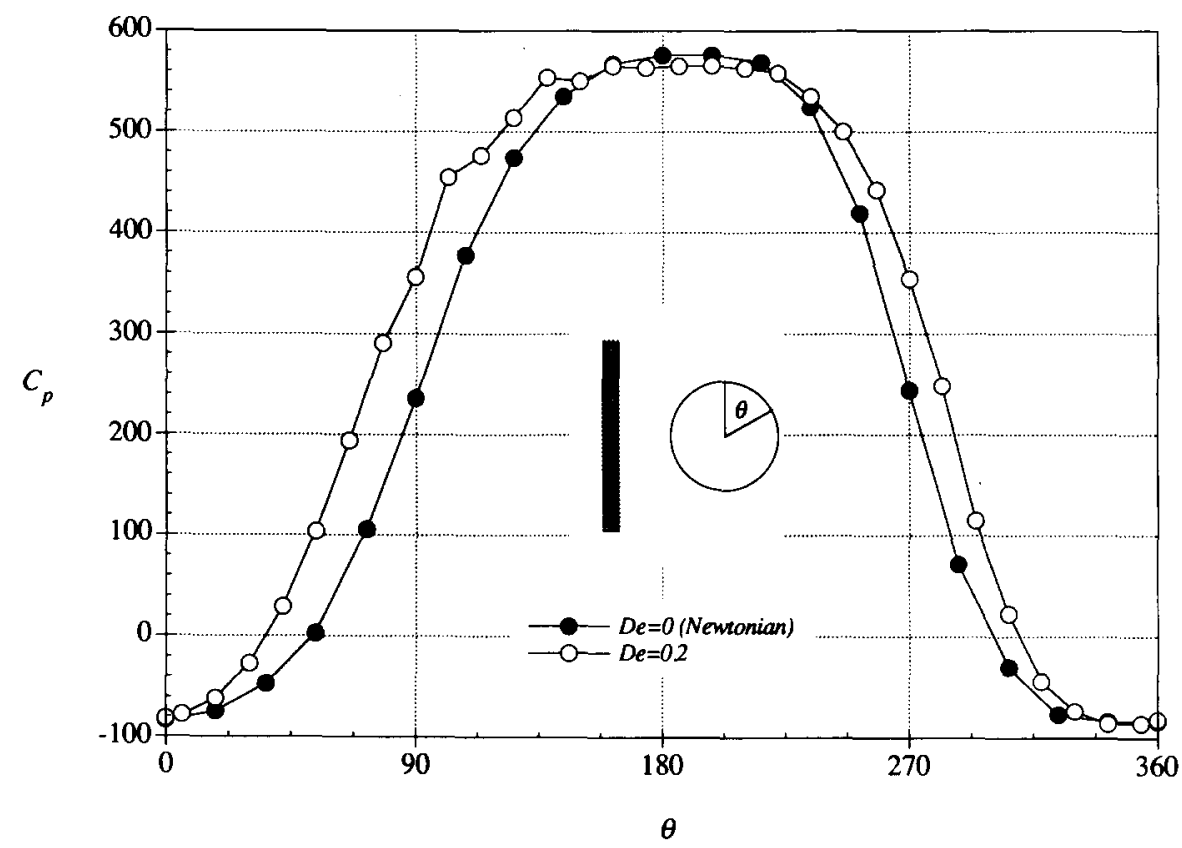

Fig. 14. Comparison between the pressure distributions in a Newtonian and a viscoelastic fluid. $R e=0.05, L / a=5$, $y / a=2$. 


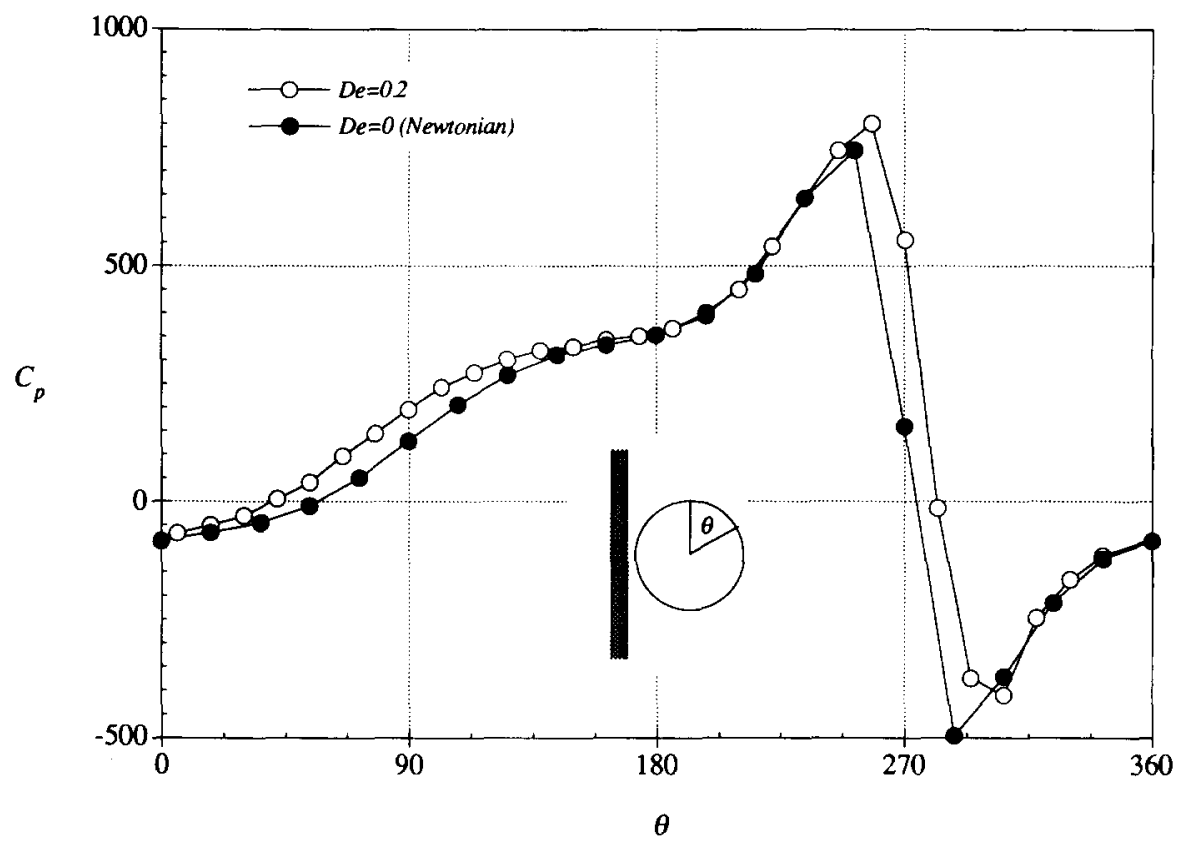

Fig. 15. Comparison between the pressure distributions in a Newtonian and a viscoelastic fluid. $R e=0.05, L / a=5$, $y / a=1.2$.

\subsubsection{The horizontal configuration}

We will consider the interaction of two particles settling abreast. Joseph et al. [8] reported that two spheres released side-by-side in a polymer solution attract and approach each other if their initial separation is not too large. This is in clear contrast to the inertial repulsion well documented for Newtonian flows (Jayaweera and Mason [30]). We again use static and dynamic simulations to study the particle-particle interaction in this configuration. The two particles are fixed or released symmetrically across the centerline of the channel with a center-to-center distance of $s$. The width of the channel is $L=20 a$ for static calculations.

The lateral force from static calculations is shown in Fig. 19. A positive lift on the left particle implies attraction. For a Newtonian fluid at $R e=0.05$, the two particles repel each other when they are close. This repulsion force diminishes when their separation increases and eventually gives way to an "attraction" force at $s / a=10$. This bogus attraction is actually a result of repulsion from side walls; in this configuration the particles are much closer to the nearby wall than they are from each other. If inertia is removed but normal stress introduced in the calculation $(R e=0, D e=1)$, the inter-particle force is reversed. A strong attraction force exists between the particles, and it decreases as the particles become farther apart. Eventually the attraction is replaced by a "repulsion", which is probably caused by wall attraction. When inertia and normal stress coexist, as is the case in the experiments, the two mechanisms compete with each other and the outcome will depend on their relative strength. Fig. 19 shows a lift curve at $R e=0.05$ and $D e=1$; in this case the normal stress apparently has an upper hand.

The lateral force between two particles is very different from the force exerted on a single particle by the channel walls (see Fig. 7). Firstly, the attraction force increases as two particles 


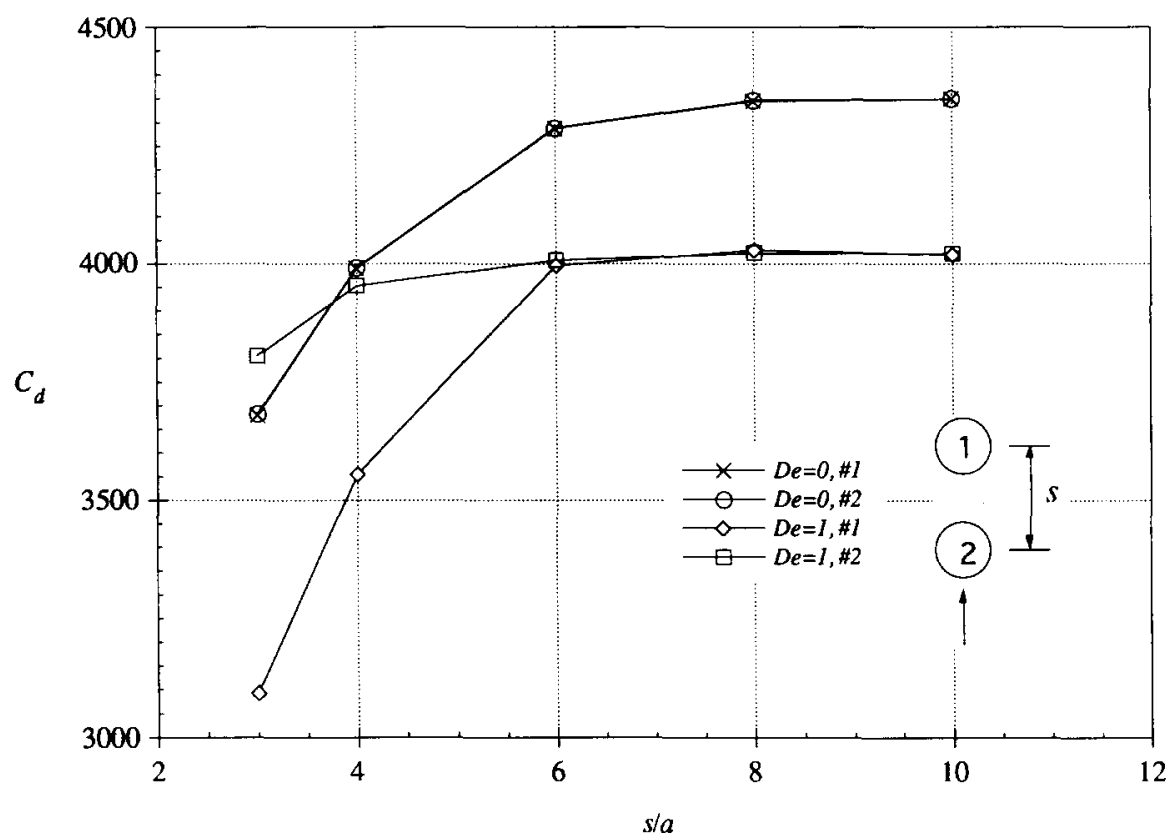

Fig. 16. The drag forces on two particles fixed tandem in space. $R e=5 \times 10^{-3}, L / a=8$.

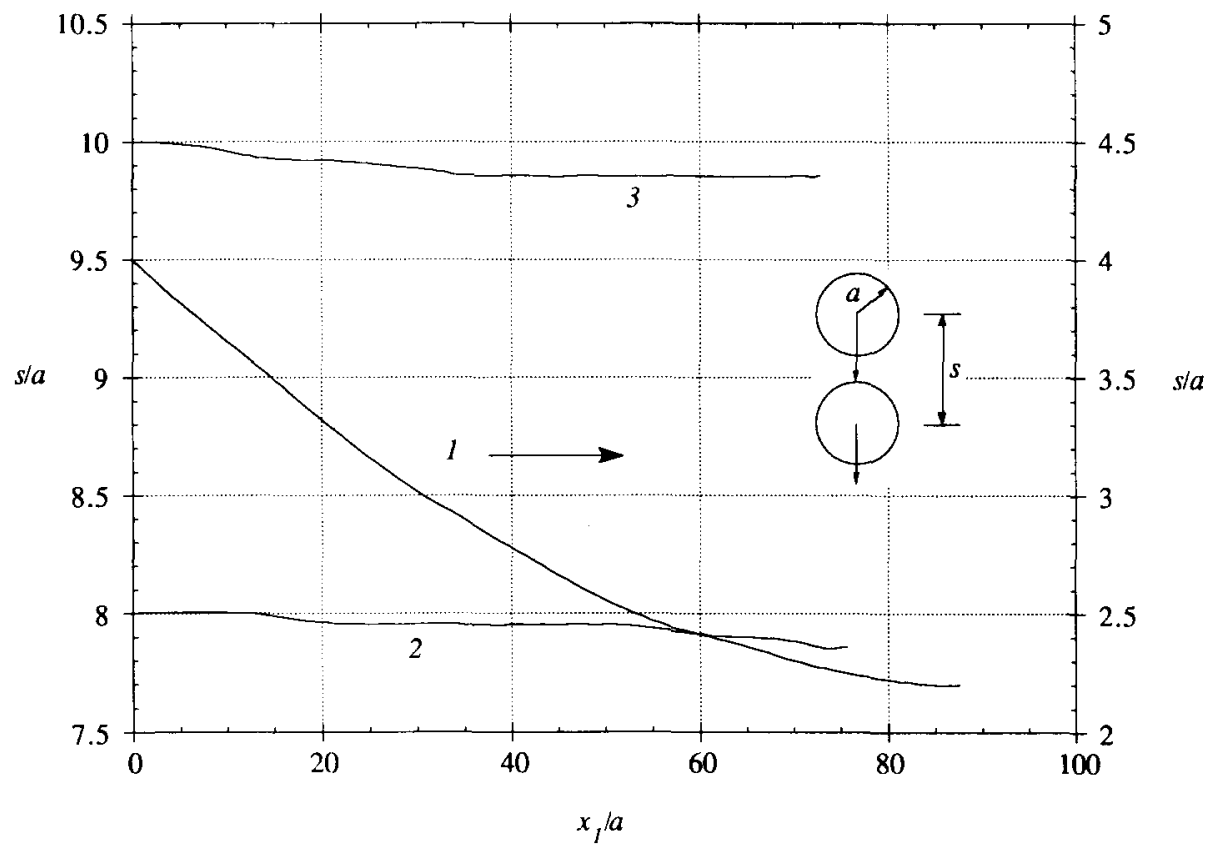

Fig. 17. The interaction of two particles falling one on top of the other. The abscissa is the vertical distance traveled by the particle on top. $L / a=8, \rho_{\mathrm{s}} / \rho_{\mathrm{f}}=1.0002, R e=0$. (1) The initial center-to-center distance is $s_{0}=4 a$. At the end of the simulation, $\mathrm{De}=0.3, \mathrm{Fr}=2.18 \times 10^{5}$; (2) $s_{0}=8 \mathrm{a}$. At the end of the simulation, $\mathrm{De}=0.21, \mathrm{Fr}=4.62 \times 10^{5}$; (3) $s_{0}=10 a$. At the end of simulation, $D e=0.21, F r=4.62 \times 10^{5}$. 

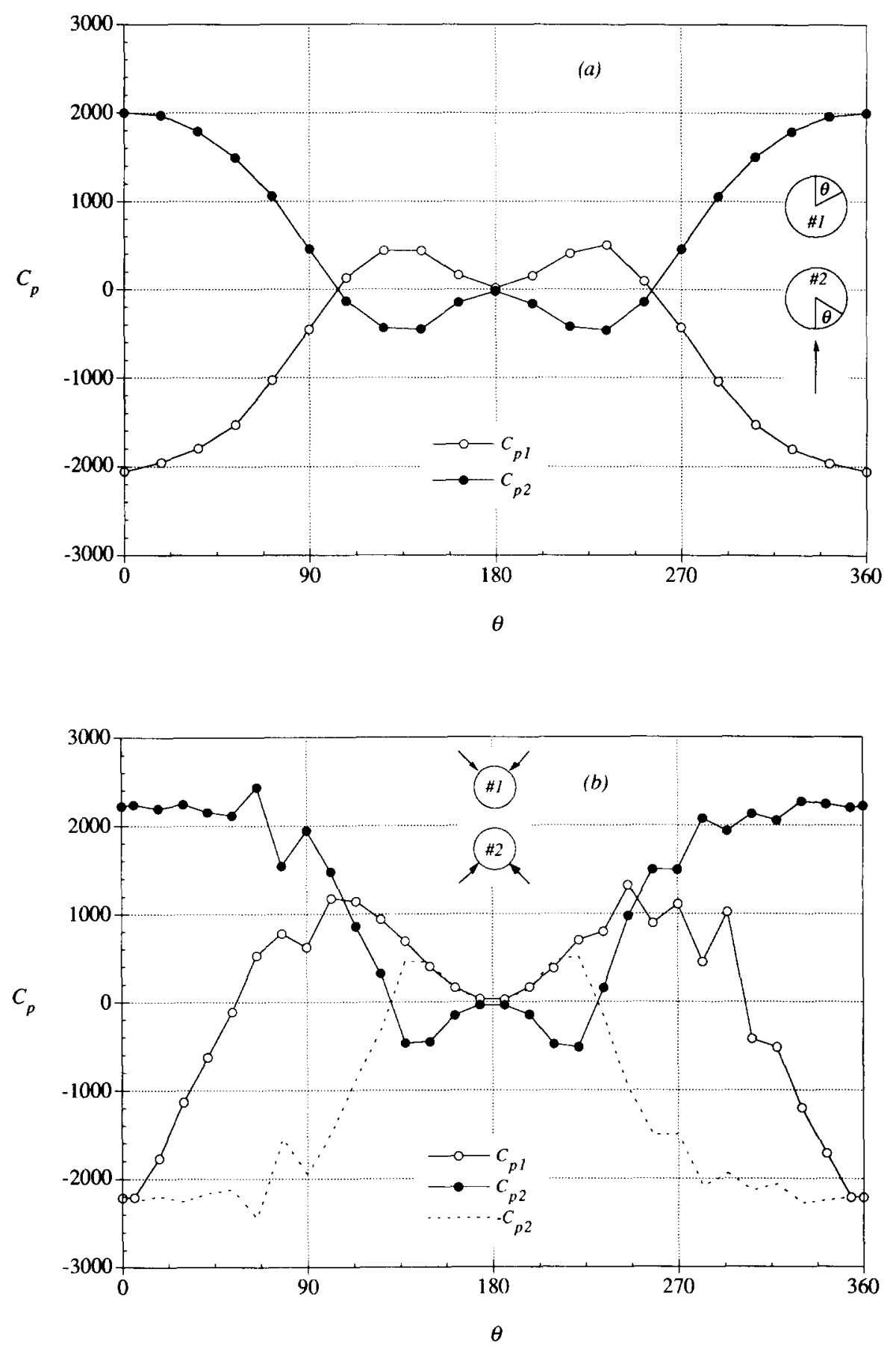

Fig. 18. The pressure distributions on two particles settling tandem. $L / a=8, s / a=3, R e=5 \times 10^{-3}$. The pressure is set to zero at the midpoint between the two particles, and $\theta$ is defined in opposite senses on them. (a) $D e=0$ (Newtonian fluid); (b) $D e=1$. The kinks in the curves are caused by numerical errors and can be suppressed by refining the mesh around the particles. The dashed line is the mirror image of $C_{\mathrm{p} 2}$. 


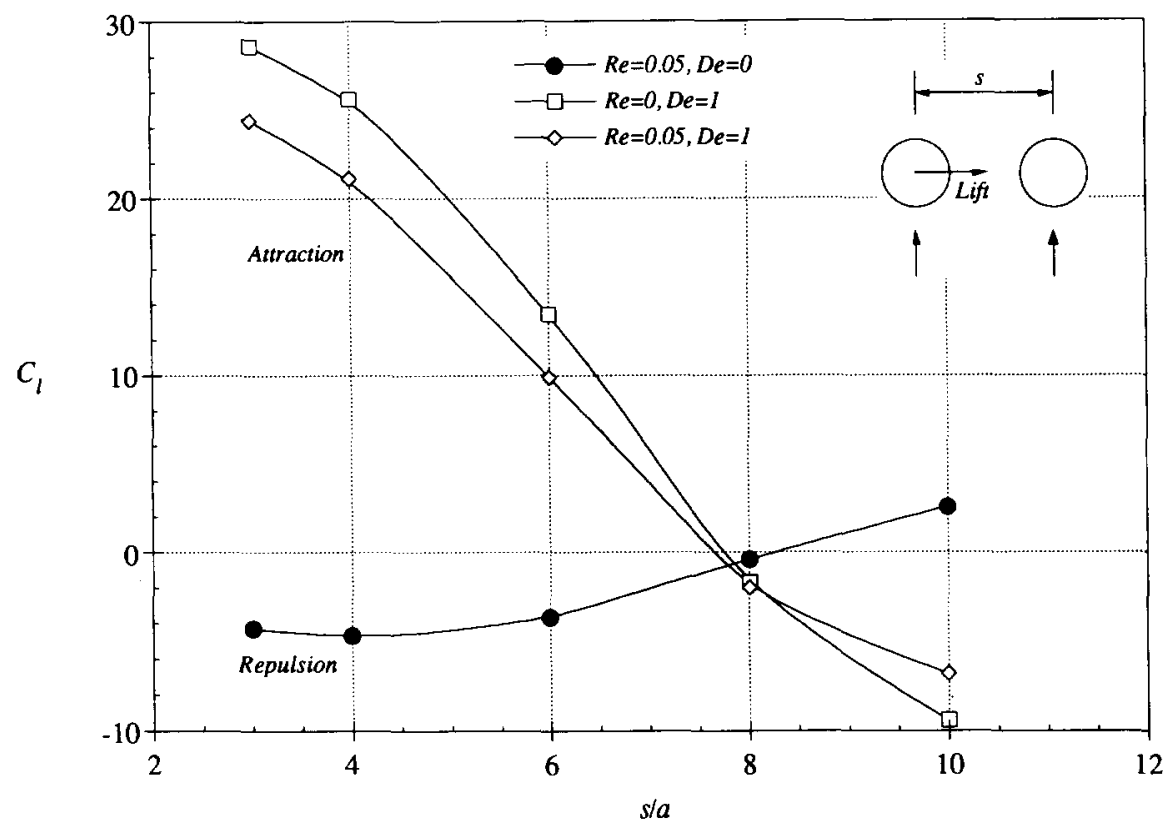

Fig. 19. Inter-particle forces on a pair of particles fixed side by side in a channel. A Newtonian lift curve is also shown for comparison. $L / a=20$.

get closer and no repulsion force is discovered. Secondly, the wall attraction arises only when the channel is sufficiently narrow. The particle-particle attraction, on the other hand, does not vanish when the channel width increases (Fig. 20). If the channel is relatively narrow, wall attractions will cancel part of the inter-particle attraction, resulting in a smaller lateral force (e.g., $L / a=10$ ). As the walls move away, the lateral force seems to tend to a limiting value, which is the particle-particle attraction in the absence of bounding walls. The comparison between Figs. 10 and 20 is interesting because for Newtonian fluids, particle-wall interactions are similar to particle-particle interactions.

For reasons given before, the fluid inertia is put to zero in dynamic simulations of two circular particles settling abreast. To reduce computational cost, we use a channel of $L=10 a$, which is narrower than that used in the static computation. This is expected to have only qualitative effects on the behavior of the particles (see Fig. 20). Two simulations are shown in Fig. 21.

Immediately after the particles are dropped, they tend to repel each other and move apart. This initial transient is similar to that observed in Fig. 17. After that, the particles start to attract and approach each other, rotating in the mean time as if rolling up the vertical plane of symmetry between them. When the two particles are close enough, they behave like a single long particle, and as such begin to turn the line of centers toward the direction of settling. For this configuration, the inter-particle attraction in Fig. 16 kicks in. The two particles will eventually touch and fall as a long particle. The initial center-to-center distance has a subtle effect on the scenario. For smaller $s_{0}$ (Fig. 21(a)), the lateral approaching occurs first and the turning of the doublet follows. If $s_{0}$ is relatively large (Fig. 21(b)), the turning and approaching happen simultaneously. When the doublet is slanted during the turning, it also drifts sideways as in a 


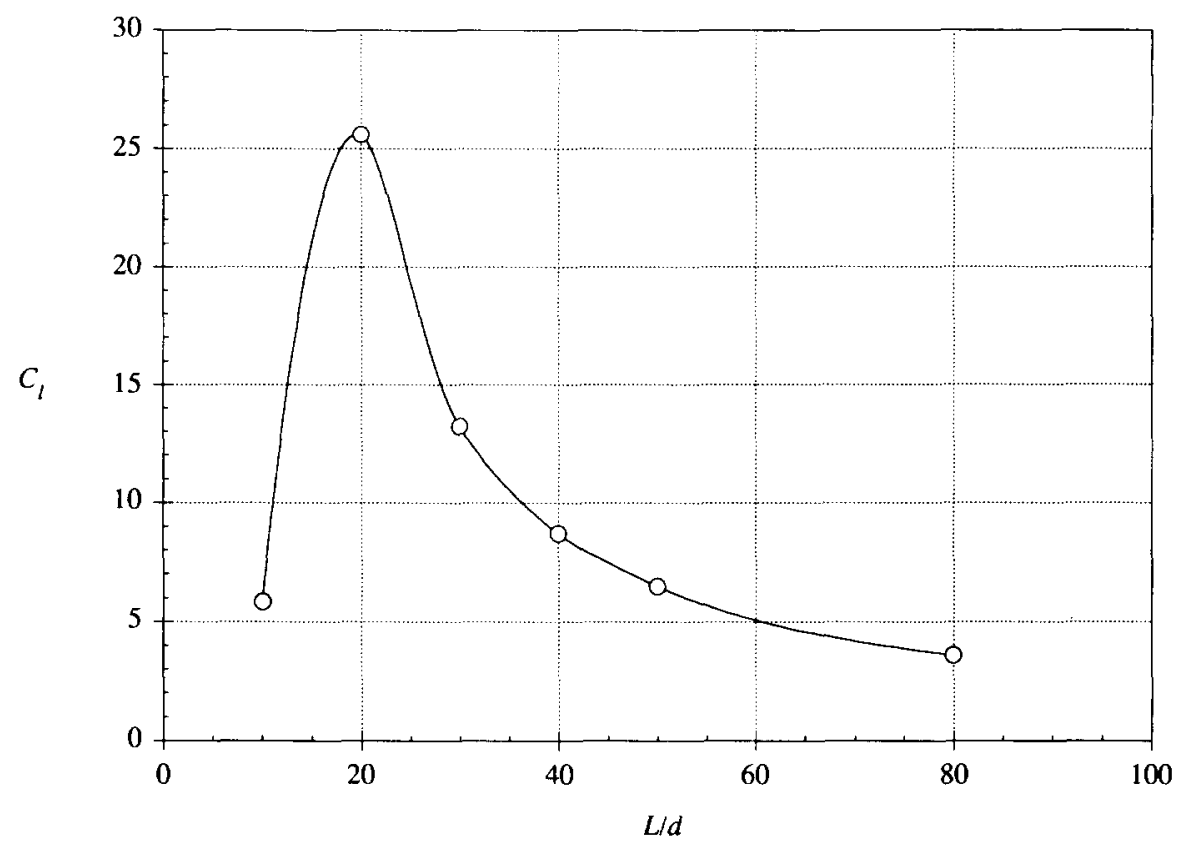

Fig. 20. Wall effects on the particle-particle interaction. The separation between the two particles is fixed at $s / a=4$. $R e=0, D e=1$.

Newtonian fluid. At the end of both simulations, the doublet has come close to one side wall and wall effects start to interfere. Though the dynamic simulations in two dimensions cannot be rigorously compared to the experiments [8], they have correctly reproduced the qualitative features observed.

The lateral attraction between two particles settling side by side results from a pressure distribution that bears the signature of viscoelasticity. Fig. 22 compares the pressure distribution on the left particle in a Newtonian and viscoelastic fluid. The pressure on the sides of the particle (near $\theta=90^{\circ}$ and $270^{\circ}$ ) is elevated by viscoelasticity. But the pressure increase is much higher on the outside of the particle $\left(\theta \approx 90^{\circ}\right)$, thus giving rise to a lateral force pulling the two particles together. One may note the similarity between Fig. 22 and Fig. 14.

\section{Conclusions}

This paper investigates the viscoelastic effects on the sedimentation of particles in the presence of solid walls or another particle. The non-linear effects of inertia in these situations are well known from previous studies. The main results of this paper may be summarized as follows.

(a) For a particle settling in a vertical channel, viscoelasticity generates a wall repulsion if the particle is very close to the wall and a wall attraction if they are farther apart. The particle will approach an eccentric equilibrium position which depends on the Reynolds and Deborah numbers. 

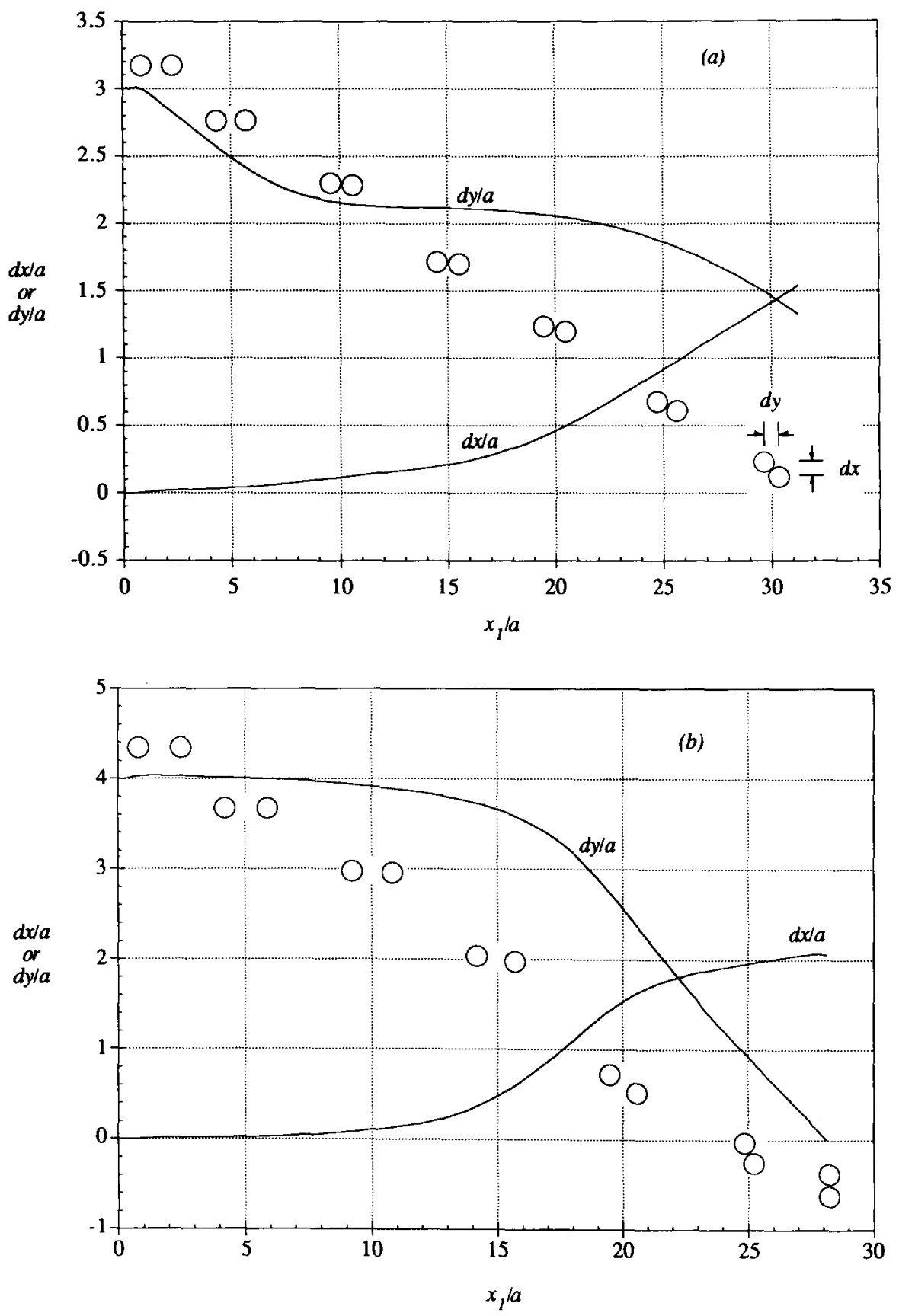

Fig. 21. The interaction between a pair of particles released side by side. $L / a=10, \rho_{\mathrm{s}} / \rho_{\mathrm{f}}=1.0005, R e=0$. (a) Initial separation $s_{0}=3 a$. At the end of the simulation $D e \approx 1.8, F r \approx 1.36 \times 10^{4}$ for both particles. (b) Initial separation $s_{0}=4 a$. At the end of the simulation $D e \approx 1.43, F r \approx 2.17 \times 10^{4}$ for both particles. 


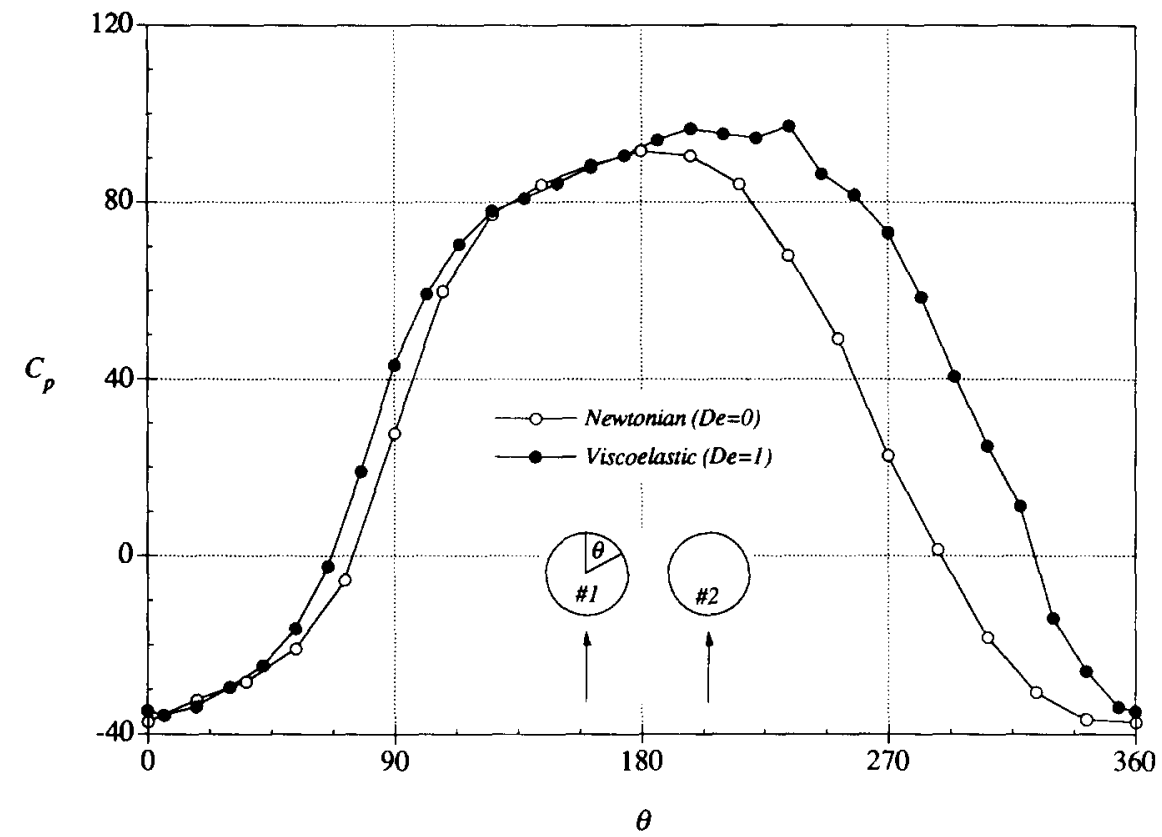

Fig. 22. Comparsion of the pressure distributions on the left particle in a Newtonian and a viscoelastic fluid. $L / a=20$, $\operatorname{Re}=0.05, s / a=3$.

(b) The wall attraction is a result of both walls and it vanishes when the channel is sufficiently wide.

(c) Two particles settling one on top of the other attract and approach each other if their initial separation is not too large.

(d) Two particles settling side by side attract and approach each other. The doublet rotates till the line of centers is aligned with the direction of fall.

(e) Viscoelasticity affects the motion of particles by modifying the pressure distribution on them. The direct contribution of normal stresses to the force and torque is unimportant.

Some of the numerical results (items (c) and (d)) agree with experiments very well, while others seemingly do not. For instance, the wall repulsion has not been documented in experiments, and the separation of two particles released one far above the other is not realized in dynamic simulations. These discrepancies may have to do with the two-dimensionality of the current study.

\section{Acknowledgements}

This work was partially supported by a NSF Grand Challenge HPCC grant (ECS 9527123), by the US Army, Mathematics and AHPCRC, by the DOE, Department of Basic Energy Sciences and by the Minnesota Supercomputer Institute. We are deeply indebted to Professor M.J. Crochet who allowed us access to POLYFLOW and made many suggestions. We also 
thank V. Legat and C. Bodart for sharing their work and helping with the code. J.F. was partly supported by a Doctoral Dissertation Fellowship from the Graduate School, University of Minnesota.

\section{References}

[1] L.G. Leal, The motion of small particles in non-Newtonian fluids, J. Non-Newtonian Fluid Mech., 5 (1979) $33-78$.

[2] L.G. Leal, The slow motion of slender rod-like particles in a second-order fluid, J. Fluid Mech., 69 (1975) $305-337$.

[3] Y.J. Liu and D.D. Joseph, Sedimentation of particles in polymer solutions, J. Fluid Mech., 255 (1993) 565-595.

[4] A. Karnis and S.G. Mason, Particle motions in sheared suspensions. XIX. Viscoelastic media, Trans. Soc. Rheol., 10 (1966) 571-592.

[5] F. Gauthier, H.L. Goldsmith and S.G. Mason, Particle motions in non-Newtonian media I. Couette flow, Rheol. Acta, 10 (1971) 344-364.

[6] F. Gauthier, H.L. Golsdmith and S.G. Mason, Particle motions in non-Newtonian media II. Poiseuille flow, Trans. Soc. Rheol., 15 (1971) 297-330.

[7] M.J. Riddle, C. Narvaez and R.B. Bird, Interactions between two spheres falling along their line of centers in a viscoelastic fluid, J. Non-Newtonian Fluid Mech., 2 (1977) 23-35.

[8] D.D. Joseph, Y.J. Liu, M. Poletto and J. Feng, Aggregation and dispersion of spheres falling in viscoelastic liquids, J. Non-Newtonian Fluid Mech., 54 (1994) 45-86.

[9] Y.J. Liu, J. Nelson, J. Feng and D.D. Joseph, Anomalous rolling of spheres down an inclined plane, J. Non-Newtonian Fluid Mech., 50 (1993) 305-329.

[10] R.I. Tanner, Observations on the use of Oldroyd-type equations of state for viscoelastic liquids, Chem. Eng. Sci., 19 (1964) 349-355.

[11] W.M. Jones, A.H. Price and K. Walters, The motion of a sphere falling unter gravity in a constant-viscosity elastic liquid, J. Non-Newtonian Fluid Mech., 53 (1994) $175-196$.

[12] P. Brunn, The motion of rigid particles in viscoelastic fluids, J. Non-Newtonian Fluid Mech., 7 (1980) 271-288.

[13] B.P. Ho and L.G. Leal, Migration of rigid spheres in a two-dimensional unidirectional shear flow of a second-order fluid, J. Fluid Mech., 76 (1976) 783-799.

[14] P.C.-H. Chan and L.G. Leal, A note on the notion of a spherical particle in a general quadratic flow of a second-order fluid, J. Fluid Mech., 82 (1977) 549-559.

[15] P. Brunn, Interaction of spheres in a viscoelastic fluid, Rheol. Acta, 16 (1977) 461-465.

[16] B. Caswell, The stability of particle motion near a wall in Newtonian and non-Newtonian fluids, Chem. Eng. Sci., 27 (1972) 373-389.

[17] K. Walters and R.I. Tanner, The motion of a sphere through an elastic fluid, in P.R. Chhabra and D. De Kee (Eds.), Transport Processes in Bubbles, Drops and Particles, Hemisphere, 1992, pp. 73-86.

[18] C. Bodart and N.J. Crochet, The time-dependent flow of a viscoelastic fluid around a sphere, J. Non-Newtonian Fluid Mech., 54 (1994) 303-329.

[19] L.E. Becker, G.H. Mckinley, H.K. Rasmussen and O. Hassager, The unsteady motion of a sphere in a viscoelastic fluid, J. Rheol., 38 (1994) 377-403.

[20] J. Feng, H.H. Hu and D.D. Joseph, Direct simulation of initial value problems for the motion of solid bodies in a Newtonian fluid. Part. 1. Sedimentation, J. Fluid Mech., 261 (1994) 95-134.

[21] J. Feng, H.H. Hu and D.D. Joseph, Direct simulation of initial value problems for the motion of solid bodies in a Newtonian fluid. Part. 2. Couette and Poiseuille flows, J. Fluid Mech., 277 (1994) 271-301.

[22] F. Debae, V. Legat and M.J. Crochet, Practical evaluation of four mixed finite element methods for viscoelastic flow, J. Rheol., 38 (1994) 421-442.

[23] P.Y. Huang and J. Feng, Wall effects on the flow of viscoelastic fluids around a circular cylinder, J. Non-Newtonian Fluid Mech., 60 (1995) 179-198. 
[24] B. Khomami, K.K. Talwar and H.K. Ganpule, A comparative study of higher- and lower-order finite element techniques for computation of viscoelastic flows, J. Rheol., 38 (1994) 225-289.

[25] E.O.A. Carew and P. Townsend, Slow visco-elastic flow past a cylinder in a rectangular channel, Rheol. Acta, 30 (1991) 58-64.

[26] S.A. Dhahir and K. Walters, On non-Newtonian flow past a cylinder in a confined flow, J. Rheol., 33 (1989) 781-804.

[27] A.S. Dvinsky and A.S. Popel, Motion of a rigid cylinder between parallel plates in Stokes flow. Part 1. Motion in a quiescent fluid and sedimentation, Compu. Fluids, 15 (1987) 391-404.

[28] W.Y. Wang, J. Feng and D.D. Joseph, A numerical study of sphere-wall and sphere-sphere interactions in slow flow of non-Newtonian fluids, in preparation.

[29] J. Feng, D.D. Joseph, R. Glowinski and T.W. Pan, A three-dimensional computation of the force and torque on an ellipsoid settling slowly through a viscoelastic fluid, J. Fluid Mech., 283 (1995) 1-16.

[30] K.O.L.F. Jayaweera and B.J. Mason, The behavior of freely falling cylinders and cones in a viscous fluid, J. Fluid Mech., 22 (1965) 709-720. 